\title{
Superintegrable Systems on Moduli Spaces of Flat Connections
}

\author{
S. Arthamonov ${ }^{1,2}$ (]) N. Reshetikhin ${ }^{1,3,4}$ \\ 1 Department of Mathematics, University of California, Berkeley, Berkeley, CA 94720, USA. \\ E-mail: artamonov@berkeley.edu; reshetik@math.berkeley.edu \\ 2 ITEP, Moscow, Russia \\ 3 Physics Department, St. Petersburg University, Saint Petersburg, Russia \\ $4 \mathrm{KdV}$ Institute for Mathematics, University of Amsterdam, Science Park 904, Amsterdam 1098 XH, \\ The Netherlands
}

Received: 1 October 2019 / Accepted: 25 May 2021

Published online: 31 July 2021 - (C) The Author(s) 2021

\begin{abstract}
The main result of this paper is the construction of a family of superintegrable Hamiltonian systems on moduli spaces of flat connections on a principal $G$-bundle on a surface. The moduli space is a Poisson variety with Atiyah-Bott Poisson structure. Among particular cases of such systems are spin generalizations of RuijsenaarsSchneider models.
\end{abstract}

\section{Introduction}

Let $G$ be a connected simple linear algebraic group over $\mathbb{C}$. In this paper we construct superintegrable Hamiltonian systems on moduli spaces of flat $G$-connections over any oriented surface with nonempty boundary. Hamiltonians of such systems are traces of holonomies along non intersecting non self-intersecting curves. The construction naturally works in the same way for various real forms of $G$, for example for compact simple Lie groups or for split real forms.

The fact that Hamiltonian integrable systems appear in the context of gauge theories is not new, with Hitchin systems [H87] being one of the well known examples. Calogero-Moser type systems were put in the context of gauge theory in early 1990's, see [GN94, GN95] and references therein. ${ }^{1}$ At about the same time, Jeffrey and Weitsman [JW92,JW94] have constructed an integrable system on the $S U(2)$ moduli space of flat connections on a closed genus $g$ surface. Hamiltonians of this system were given by traces of holonomies along the maximal collection of nonintersecting curves. In the higher rank case, the total number of hamiltonians which can be constructed from class functions of such holonomies is insufficient for Liouville integrability.

The main result of this paper involves the notion of a superintegrable Hamiltonian system which we review in Sect. 1, and of graph functions which we review in Sect. 2.2. Denote the moduli space of flat $G$-connections on $\Sigma$ as $\mathcal{M}_{\Sigma}^{G}$. Our main result is the following theorem.

\footnotetext{
${ }^{1}$ See also [FK13,AO19] for more recent devolopments and references.
} 
Theorem 1. Let $\Sigma$ be an oriented surface of genus $g$ with $b>0$ boundary components and $G$ be a connected simple linear algebraic group over $\mathbb{C}$. For each disjoint union of pairwise nonhomotopic simple closed curves $C=C_{1} \sqcup \cdots \sqcup C_{k}$ in $\Sigma$, none of which is homopotic to a boundary component, the following inclusion of Poisson algebras defines an affine superintegrable system

$$
Z_{\partial \Sigma} \subset B_{C} \subset J_{\Sigma \backslash C} \subset \mathcal{O}\left[\mathcal{M}_{\Sigma}^{G}\right]
$$

Here $Z_{\partial \Sigma}$ is the algebra of $G$-invariant functions on holonomies around boundary components, $B_{C}$ is a subalgebra of the coordinate ring $O\left[\mathcal{M}_{\Sigma}^{G}\right]$ generated by graph functions $F_{\Gamma, V, c}$ with $\Gamma$ being contractible to $C \sqcup \partial \Sigma$. Similarly, $J_{\Sigma \backslash C}$ is a subalgebra generated by graph functions $F_{\Gamma, V, c}$ with $\Gamma \subset(\Sigma \backslash C)$.

We also introduce the notion of a refinement of one superintegrable systems by the other. The refinement defines a partial order on the space of superintegrable systems.

For a torus with one puncture these systems are spin Ruijsenaars systems (also known as relativistic spin Calogero-Moser systems) [FGNR00, Res16]. For a torus with two punctures they are relativistic deformations of two sided spin Calogero-Moser systems [Res18] corresponding to symmetric pair $G \subset G \times G$ where $G$ is embedded diagonally. A closely related example of systems on a torus with $n$ punctures and rank one conjugation orbits of $S L_{n}$ is closely related to the systems studied in [CF18]. In this case the systems are actually Liouville integrable and they admit a superintegrable refinement. The maximal integrability in this case is achieved by a superintegrable system with $r=\operatorname{rk}(G)$ independent Poisson commuting integrals. The minimal integrability is a Liouville integrability with $b \operatorname{rk}(G)$ Poisson commuting integrals. In our framework these correspond to different choices of cycles $C$.

The mapping class group acts on the moduli space of flat connections by Poisson automorphisms. If $C$ and $C^{\prime}$ are collections of cycles on surface $\Sigma$ which belong to the same orbit of the Mapping Class Group action, the corresponding superintegrable systems are isomorphic. An example of such isomorphism in the genus one case for involution exchanging the equator and meridian of the torus gives rise to a self-duality of the Ruijsenaars system [FGNR00,FR99,FK11]. For a spin version of this duality see [Res16].

The plan of this paper is as follows. In the first section we recall the definition of superintegrable (degenerately integrable) systems. There we also define the notion of an affine superintegrable system in the algebro-geometrical setting. The second section is an overview of basic notions about moduli spaces of flat connections on a surface. In this section we also recall the definition of graph functions and the description of Poisson brackets between two such functions. In the third section we describe the main result, the construction of a family of Hamiltonian systems defined by a choice of non intersecting, non self intersecting cycles and prove their superintegrability. At the end of this section we introduce the notion of a partial order on such systems. In section 4 we explain how solutions to equations of motion of these superintegrable systems can be solved using the projection method. Sect. 5 has some genus one examples. In the conclusion we define a conjecturally superintegrable system on the space of chord diagrams, discuss the case of non-generic conjugation orbits and some further directions. 


\section{Superintegrable Systems}

1.1. Superintegrable systems. For an overview of superintegrable systems see for example [SInt, Res16]. Here we recall briefly basic definitions and introduce the notion of an affine superintegrable system.

The notion of a superintegrable Hamiltonian systems was introduced in [Nek72] (where he called them degenerate integrable systems) as a generalization of the Liouville integrable systems. Superintegrable system on a $2 n$ dimensional smooth symplectic manifold has $m>n$ independent ${ }^{2}$ first integrals, however only $k$ of them are in involution and $m+k=2 n$. A particular case when $m=k=n$ corresponds to Liouville integrable systems. First examples of such systems appeared earlier [Pa26,F35,FMSW65]. A family of examples of superintegrable systems associated to Lie groups was given in [MF78]. More recent examples include charateristic systems on simple Poisson-Lie groups [Res03a], spin Calogero-Moser and Ruijsenaars systems [Res03b] and their relativistic counterparts [Res16].

Geometrically, a superintegrable system of rank $k$ on a symplectic manifold $\mathcal{M}_{2 n}$ consists of a Poisson manifold $\mathcal{P}_{2 n-k}$, a manifold $\mathcal{B}_{k}{ }^{3}$ (considered as a Poisson manifold with trivial symplectic structure) and Poisson projections

$$
\mathcal{M}_{2 n} \stackrel{p_{1}}{\longrightarrow} \mathcal{P}_{2 n-k} \stackrel{p_{2}}{\longrightarrow} \mathcal{B}_{k}
$$

Denote by $A=C^{\infty}\left(\mathcal{M}_{2 n}\right)$ the algebra of smooth functions on $\mathcal{M}_{2 n}$. It is equipped with a Poisson bracket $\{$,$\} determined by the symplectic structure. In terms of functions$ on $\mathcal{M}_{2 n}$ superintegrable system is determined by two Poisson subalgebras of $A$ :

$$
B \subset J \subset A
$$

where $J=C^{\infty}\left(\mathcal{P}_{2 n-k}\right)$ and $B=C^{\infty}\left(\mathcal{B}_{k}\right)$. The subalgebra $B$ is a Poisson commutative subalgebra and $J$ is the Poisson centralizer of $B$, i.e. $J$ is the maximal subalgebra in $A$ such every element from $J$ Poisson commutes with every element from $B$.

Elements of subalgebra $B$ define derivations of $A$

$D_{b}: A \rightarrow A, \quad D_{b}(a)=\{b, a\}, \quad D_{b}\left(a_{1} a_{2}\right)=a_{1} D_{b}\left(a_{2}\right)+D_{b}\left(a_{1}\right) a_{2}, \quad b \in B, a, a_{1}, a_{2} \in A$.

Because $B$ is Poisson commutative, these derivations commute

$$
D_{b_{1}} D_{b_{2}}-D_{b_{2}} D_{b_{1}}=0
$$

Derivation $D_{b}$ is the Lie derivative along the Hamiltonian vector field on $\mathcal{M}_{2 n}$ generated by $b$. We refer to elements of $B$ as Hamiltonians of a superintegrable system (2). On the other hand, elements of subalgebra $J$ are precisely the first integrals of the Hamilton flow generated by $b$, i.e. they are constant on flow lines of the Hamiltonian vector field generated by $b$.

Fix $b_{1}, \ldots, b_{k} \in B$, a choice of independent Hamiltonians. Corresponding vector fields $D_{b_{1}}, \ldots, D_{b_{k}} \in T \mathcal{M}_{2 n}$ are independent at every point $x \in \mathcal{M}_{2 n}$ and hence form a basis on the $k$-dimensional subspace of $T_{x} \mathcal{M}_{2 n}$ defined by the level set of $J$. Algebraically, these vector fields $D_{b_{1}}, \ldots, D_{b_{k}} \in \operatorname{Der}_{J}(A)$ define derivations of $A$ relative to $J$. Balance of dimensions in (1) is equivalent to the fact that $\operatorname{Der}_{J}(A)$ is generated as an $A$-module by Hamiltonian vector fields of the form $D_{b}$ for $b \in B$.

\footnotetext{
2 On a smooth manifold $\mathcal{M}$ (complex or real) we call functions $f_{1}, \ldots, f_{k} \in C^{\infty}(\mathcal{M})$ independent if the corresponding differentials $\mathrm{df}_{1}, \ldots, \mathrm{df}_{k} \in \Gamma\left(T^{*} \mathcal{M}\right)$ are linearly independent at every point in $\mathcal{M}$.

${ }^{3}$ Typically $\mathcal{B}_{k}$ is not smooth but stratified by smooth strata with one smooth higher dimensional stratum. It is frequently an orbifold.
} 
1.2. Affine superintegrable systems. In many interesting examples superintegrable systems appear in families on affine Poisson varieties, we refer to such families as affine superintegrable systems.

Definition 2. Let $\mathcal{A}$ be an affine variety ${ }^{4}$ over $\mathbb{C}$ with a ring of regular functions $A=$ $\mathcal{O}[\mathcal{A}]$ equipped with a Poisson bracket $\{\}:, A \otimes A \rightarrow A$. Suppose further that the Poisson tensor has rank $2 n$ on an open dense subset of $\mathcal{A}$. We say that a chain of inclusions of finitely generated Poisson subalgebras

$$
Z \subset B \subset J \subset A
$$

is an affine superintegrable system if

$$
\{Z, A\}=0=\{B, J\}
$$

and

$$
\operatorname{dim} \mathcal{B}+\operatorname{dim} \mathcal{J}-2 \operatorname{dim} \mathcal{Z}=2 n=\operatorname{dim} \mathcal{A}-\operatorname{dim} \mathcal{Z}
$$

where $\mathcal{J}=\operatorname{Spec} J, \mathcal{B}=\operatorname{Spec} B, \mathcal{Z}=\operatorname{Spec} Z$ stand for the spectrum of prime ideals of $J, B, Z$ respectively.

Inclusions (4) are equivalent to the chain of dominant maps of affine schemes which preserve the Poisson bracket

$$
\mathcal{A} \stackrel{p_{1}}{\longrightarrow} \mathcal{J} \stackrel{p_{2}}{\longrightarrow} \mathcal{B} \stackrel{p_{3}}{\longrightarrow} \mathcal{Z} \text {. }
$$

Definition 2 implies that general fibers of $p_{2}$ are symplectic leaves of $\mathcal{J},{ }^{5}$ while general fibers of the composition map $p$

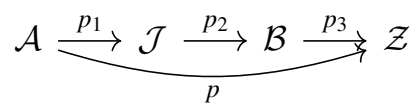

are symplectic leaves of $\mathcal{A}$.

As a corollary, open subset $\mathcal{M}_{2 n}(z)$ of irreducible component of a general fiber $p^{-1}(z), z \in \mathcal{Z}$ can be viewed as a phase space of a superintegrable system. $\mathcal{M}_{2 n}(z)$ comes equipped with two poisson projections of the form (1). Thus, as we already mentioned, affine superintegrable systems should be regarded as families of superintegrable systems.

\footnotetext{
${ }^{4}$ More generally, one can take $\mathcal{A}=\operatorname{Spec} A$ to be an integral affine scheme.

${ }^{5}$ More precisely, $p_{2}^{-1}(b), b \in \mathcal{B}$ is an algebraic subset of $\mathcal{J}$, it contains an irreducible component of maximal dimension which is an affine Poisson variety. For a general $b$, this variety is equipped with a Poisson bracket of maximal rank equal to the dimension.
} 


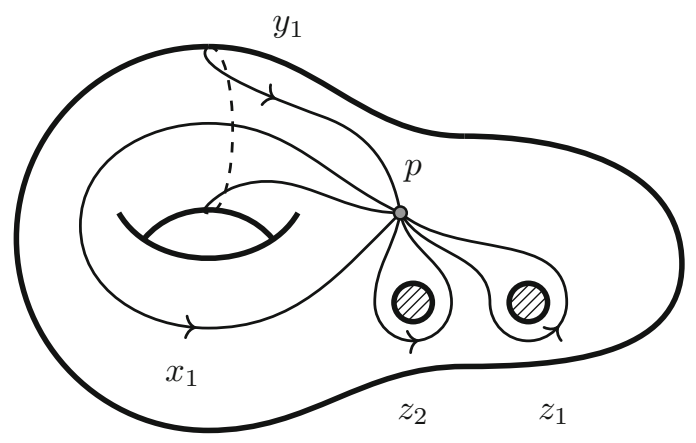

Fig. 1. One choice of arcs corresponding to generators of $\pi_{1}\left(\Sigma_{1,2}, p\right)$

1.3. Refinement of a superintegrable system. Here we will introduce the notion of a refinement of a superintegrable system. It gives a partial order on all superintegrable systems on a given Poisson algebra $A$. A minimal object in this partial order is known as a maximally superintegrable system. Maximal superintegrability means that all invariant tori are one dimensional [SInt].

Let

$$
Z \subset B_{1} \subset J_{1} \subset A, \quad Z \subset B_{2} \subset J_{2} \subset A
$$

be two superintegrable systems on a Poisson algebra $A$ with the Poisson center $Z$.

Definition 3. The first superintegrable system is a refinement of the second if the following chain of Poisson inclusions hold:

$$
Z \subset B_{1} \subset B_{2} \subset J_{2} \subset J_{1} \subset A
$$

Clearly a refinement defines a partial order on the set of integrable systems.

Another important relation is an equivalence of integrable systems

Definition 4. Two integrable systems (6) are equivalent if there is a Poisson automorphism $\varphi: A \rightarrow A$ such that $J_{2}=\varphi\left(J_{1}\right)$ and $B_{1}=\varphi\left(B_{2}\right)$.

\section{Moduli Spaces of Flat Connections}

2.1. Character variety of the fundamental group. Let $\Sigma_{g, b}$ be an oriented surface of genus $g$ with $b$ boundary components. We will consider only surfaces with $b>0$. The fundamental group $\pi_{1}(\Sigma, p)$ with the base point $p$ of such surface is generated by elements $x_{1}, \cdots, x_{g}, y_{1}, \cdots, y_{g}, z_{1}, \cdots z_{b}$ with one defining relation:

$$
\pi_{1}\left(\Sigma_{g, b}\right)=\left\langle x_{1}, \ldots, x_{g}, y_{1}, \ldots, y_{g}, z_{1}, \ldots, z_{b} \mid\left[x_{1}, y_{1}\right] \ldots\left[x_{g}, y_{g}\right] z_{1} \ldots z_{b}=\operatorname{Id}_{p}\right\rangle .
$$

Here $x_{i}$ and $y_{i}$ are fundamental cycles for $\Sigma$ and $z_{i}$ is the cycle for $i$-th boundary component. ${ }^{6}$

\footnotetext{
6 Throughout the text we always assume that the composition of paths is read from right to left. For example, $\left[x_{1}, y_{1}\right] z_{1} z_{2}$ corresponds to the contractible path along the boundary of a disc in $\Sigma_{1,2}$ on Fig. 1 .
} 
The character variety of $\pi_{1}\left(\Sigma_{g, b}\right)$ in a simple complex linear algebraic group $G$ is the categorical quotient of the space of group homomorphisms from $\pi_{1}\left(\Sigma_{g, b}\right)$ to $G$ with respect to conjugations by $G^{7}$ :

$$
\mathcal{M}_{\Sigma}^{G}=\left(\pi_{1}\left(\Sigma_{g, b}\right) \rightarrow G\right) / / G:=\operatorname{Spec}\left(\mathcal{O}\left[\operatorname{Hom}\left(\pi_{1}\left(\Sigma_{g, b}\right), G\right)\right]^{G}\right)
$$

For $b>0$ we have an obvious isomorphism:

$$
\mathcal{M}_{\Sigma}^{G} \simeq G^{\times(2 g+b-1)} / / G
$$

We can choose this isomorphism as the mapping which assigns to a group homomorphism $\rho: \pi_{1}\left(\Sigma_{g, b}\right) \rightarrow G$ an element $\left(\rho\left(x_{1}\right), \ldots, \rho\left(x_{g}\right), \rho\left(y_{1}\right), \ldots, \rho\left(y_{g}\right), \rho\left(z_{1}\right), \ldots\right.$, $\left.\rho\left(z_{b-1}\right)\right) \in G^{\times(2 g+b-1)}$ and then projects it to the conjugacy class in $G^{\times(2 g+b-1)} / / G$.

2.2. Moduli space of surface graph connections. Denote by $V(\Gamma)$ and $E(\Gamma)$ the set of vertices and edges of an oriented graph $\Gamma$. Graph connection is an assignment of a parallel transport $g_{e} \in G$ to each oriented edge $e \in E(\Gamma)$.

The gauge group $G(V) \simeq G^{V(\Gamma)}=\left\{V(\Gamma) \rightarrow G, \quad v \mapsto h_{v}\right\}$ acts on a graph connection $g$ as

$$
g_{e} \mapsto h_{t(e)} g_{e} h_{s(e)}^{-1} .
$$

where $t(e)$ is the target vertex for an oriented edge $e$ and $s(e)$ is its source vertex.

Assume that $\Gamma \subset \Sigma$ is an oriented embedded graph such that $\Sigma \backslash \Gamma$ is a disjoint collection of disks and $b$ annuli corresponding to the boundary components of $\Sigma=\Sigma_{g, b}$. We will call such graphs simple. Hereinafter we allow graphs to have multiple edges and loops.

Let $F(\Gamma)$ be the set of contractible faces, i.e. the set of disks in $\Sigma_{g, b} \backslash \Gamma$. For every disk $D \in F(\Gamma)$ choose an arbitrary vertex $w_{D} \in \partial D$ and let

$$
g_{\partial D}=g_{e_{k}}^{\epsilon_{k}} \ldots g_{e_{1}}^{\epsilon_{1}}
$$

be the holonomy of graph connection along the boundary of a disk, based at $w_{D} \in \partial D$. The product of elements $g_{e_{j}}^{\epsilon_{j}} \in G$ associated to each edge $e_{j} \in \partial D$ is given according to the total order induced by the orientation of the surface and choice of $w_{D} \in \partial D$. The relative orientation $\epsilon_{j}$ is +1 when the orientations of $e_{j}$ and $\partial D$ coincide and -1 otherwise.

A graph connection over $\Gamma \subset \Sigma$ is called flat if $g_{\partial D}=1$ for all $D \in F(\Gamma)$. Note that this condition does not depend on the choice of the base vertex $w_{D} \in \partial D$. Define the space of flat graph $G$-connections $\mathcal{A}_{(\Sigma, \Gamma)}$ on $\Gamma \subset \Sigma$ as the space of such flat connections.

When $G$ is a linear algebraic group, the space of flat graph connections is an algebraic subset of $G^{\mid E(\Gamma)) \mid}$ equipped with a regular action of $G(V)$. The moduli space offlat graph connections is the categorical quotient

$$
\mathcal{M}_{(\Sigma, \Gamma)}^{G}=\mathcal{A}_{(\Sigma, \Gamma)} / / G(V):=\operatorname{Spec}\left(\mathcal{O}\left[\mathcal{A}_{(\Sigma, \Gamma)}\right]^{G(V)}\right)
$$

\footnotetext{
7 Note that despite its name, $\mathcal{M}_{\Sigma}^{G}$ is not a variety but rather an affine scheme. Throughout the text we will always think of $\mathcal{M}_{\Sigma}^{G}$ in terms of its coordinate ring, which is precisely the $G$-invariant subring of the coordinate ring of representation variety.
} 


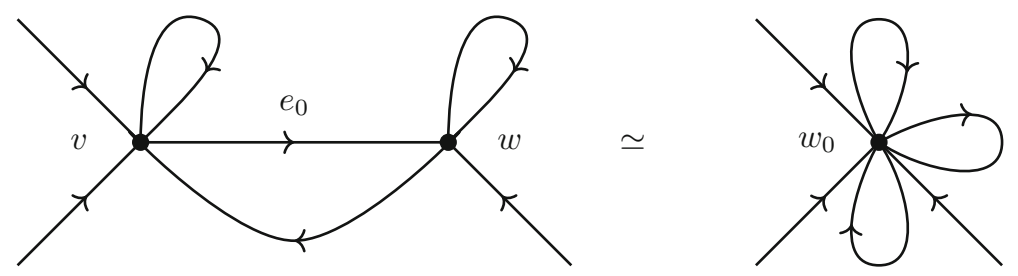

Fig. 2. Contraction of an edge

Let $e \in E(\Gamma)$ be an edge which connects two distinct vertices, denote as $\Gamma_{e}$ an embedded graph obtained from $\Gamma$ by contracting an edge $e$. Similarly, for any contractible face $D \in F(\Gamma)$ which has an edge $e \in \partial D$ that appears only once in $\partial D$ we can define an ebedded graph $\Gamma^{e}=\Gamma \backslash e$. Recall that $\Gamma$ was simple, i.e. its complement in $\Sigma$ is the union of disks and annuli. It is clear that $\left(\Sigma \backslash \Gamma^{e}\right)$ is also simple. The flatness condition $g_{\partial D}=1$ guarantees the isomorphism of schemes.

Theorem 5. The following schemes are isomorphic:

$$
\mathcal{M}_{(\Sigma, \Gamma)}^{G} \simeq \mathcal{M}_{\left(\Sigma, \Gamma_{e}\right)}^{G} \simeq \mathcal{M}_{\left(\Sigma, \Gamma^{e}\right)}^{G}
$$

Proof. Resolving one of the relations $g_{\partial D}=1$ we obtain an isomorphism of $G(V)$ modules $\mathcal{O}\left[\mathcal{A}_{(\Sigma, \Gamma)}\right] \simeq \mathcal{O}\left[\mathcal{A}_{\left(\Sigma, \Gamma^{e}\right)}\right]$. Taking the $G(V)$-invariant part we conclude

$$
\mathcal{O}\left[\mathcal{A}_{(\Sigma, \Gamma)}\right]^{G(V)} \simeq \mathcal{O}\left[\mathcal{A}_{\left(\Sigma, \Gamma^{e}\right)}\right]^{G(V)}
$$

and therefore $\mathcal{M}_{(\Sigma, \Gamma)}^{G} \simeq \mathcal{M}_{\left(\Sigma, \Gamma^{e}\right)}^{G}$.

Now let us prove the first isomorphism in (7). Let $e_{0}$ be an edge of $\Gamma$ with adjacent vertices $v=s\left(e_{0}\right)$ to $w=t\left(e_{0}\right)$. Here $s(e), t(e)$ stand for the source and target of $e$ respectively. Contracting of $e_{0}$ maps vertices $v$ and $w$ to one vertex $w_{0}$ of the new graph $\Gamma_{e_{0}}$ which is the contraction of $\Gamma$. Denote this mapping by $\pi_{e_{0}}$.

The contraction of $\Gamma$ to $\Gamma_{e_{0}}$ defines a functor between the fundamental groupoids which we will denote by the same letter $\pi_{e_{0}}: \Pi(\Gamma) \rightarrow \Pi\left(\Gamma_{e_{0}}\right)$. It is clear that this is a projection. Choose a section $\phi: \Pi\left(\Gamma_{e_{0}}\right) \rightarrow \Pi(\Gamma)$ of $\pi_{e_{0}}$, so that $\pi_{e_{0}} \phi=i d$. It is clear that $\phi$ does not have to be unique, but it always exists. This is illustrated on Fig. 2.

The functors $\pi_{e_{0}}$ and $\phi$ define the projection $p_{e_{0}}: \mathcal{A}_{(\Sigma, \Gamma)} \rightarrow \mathcal{A}_{\left(\Sigma, \Gamma_{e_{0}}\right)}$ and its section $f: \mathcal{A}_{\left(\Sigma, \Gamma_{e_{0}}\right)} \rightarrow \mathcal{A}_{(\Sigma, \Gamma)}$. Taking quotients with respect to the gauge group we obtain the projection $\left[p_{e_{0}}\right]: \mathcal{M}_{(\Sigma, \Gamma)} \rightarrow \mathcal{M}_{\left(\Sigma, \Gamma_{e_{0}}\right)}$ and its section $[f]: \mathcal{M}_{\left(\Sigma, \Gamma_{e_{0}}\right)} \rightarrow \mathcal{M}_{(\Sigma, \Gamma)}$. Comparing dimensions we conclude that these mappings are isomorphisms. The section $f$ depends on the choice of $\phi$. It is easy to see that the corresponding mapping $[f]$ between moduli spaces does not.

Corollary 6. For any oriented embedded graph $\Gamma \in \Sigma_{g, b}$ such that $\Sigma_{g, b} \backslash \Gamma$ is a disjoint union of discs and $b$ annuli, the moduli space of graph connections is isomorphic to the $G$-character variety

$$
\mathcal{M}_{\Sigma}^{G} \simeq \mathcal{M}_{(\Sigma, \Gamma)}^{G}
$$

Corollary 7. The dimensions of moduli spaces of graph connections on surface $\Sigma_{g, b}$ with $b>0$ are:

$$
\operatorname{dim}\left(\mathcal{M}_{(\Sigma, \Gamma)}^{G}\right)= \begin{cases}0 & g=0, b=1, \\ \operatorname{rk}(G) & g=0, b=2, \\ (2 g+b-2) \operatorname{dim} G & \text { otherwise }\end{cases}
$$


2.3. Colored immersed graphs. Let $\Gamma$ be an oriented graph.

Definition 8. An edge coloring of $\Gamma$ is an assignment of a finite-dimensional $G$-representation $V_{\epsilon}$ to each edge $e \in \Gamma$ of the oriented graph. We will denote edge colored graph as $(\Gamma, V)$.

We say that an oriented graph $\Gamma$ is immersed in a surface $\Sigma_{g, b}$ if the inclusion mapping $I: \Gamma \rightarrow \Sigma_{g, b}$ is locally an embedding. ${ }^{8}$ If the intersection of $\Gamma$ on $\Sigma_{g, b}$ are transversal, we will call it transversally immersed.

Orientation of the surface induces a cyclic order on edges adjacent to a given vertex. For each vertex $v \in V(\Gamma) \subset \Sigma$ let us choose a total ordering $e_{1}, \ldots, e_{n}$ of the adjacent edges which agrees with the cyclic ordering induced by the orientation of $\Sigma$. We will refer to this as edge ordering of $\Gamma \subset \Sigma$. For a finite dimensional vector space $V$ define $V^{+}=V$ and $V^{-}=V^{*}$. For each vertex of edge ordered graph define the space

$$
V(v)=V_{e_{1}}^{\epsilon_{1}} \otimes \cdots \otimes V_{e_{n}}^{\epsilon_{n}}
$$

Here $\epsilon_{i}=+1$ is oriented outward from $v$, i.e. $v=s\left(e_{i}\right)$ and $\epsilon=-1$ otherwise, i.e. when $v=t(e)^{9}$.

Definition 9. A vertex coloring of $(\Gamma, V)$ is an assignment of a vector $c_{v} \in V(v)^{G}$ to each vertex $v$ of an edge ordered oriented graph $\Gamma$.

Here $V(v)^{G}$ is the space of $G$-invariant vectors in a representation $V(v)$.

We define colored graph $\boldsymbol{\Gamma}=(\Gamma, V, c)$ as an edge ordered oriented immersed graph $\Gamma$ equipped with an edge coloring $V$ and a vertex coloring $c$.

\subsection{Invariant functions on connections associated to immersed graphs.}

2.4.1. . For each colored graph $(\Gamma, V, c)$ embedded into $\Sigma$ one can associate a function $F_{\Gamma, V, c}$ on connections on the principal $G$-bundle over $\Sigma_{g, b}$ [AMR96] as follows.

For a graph $\Gamma$ with an edge coloring $V$ define the following $G$-module

$$
\mathbf{V}(\Gamma)=\bigotimes_{v \in V(\Gamma)} V(v)
$$

Here we assume a choice of a linear ordering on vertices. The space $V(v)$ are defined in (9). Note that $V(v)$ can also be written as

$$
V(v)=\bigotimes_{e \in S(v)} V(e)^{\epsilon(e, v)}
$$

Here $S(v)$ is the star of vertex $v^{10}$ and $\epsilon(e, v)=+$ if $e$ is outgoing and $\epsilon(e, v)=-$ if it is incoming.

\footnotetext{
8 Immersed graphs may intersect on a surface and intersections does not have to be transversal.

9 Note that because the category of $G$-modules is symmetric, there exists a canonical isomorphism between $V(v)$ for different choices of edge ordering. Still, it will be convenient for us to assume that we have chosen a total order of edges adjoint to the given vertex.

10 In our case this is the set of edges adjacent to $v$.
} 
By changing the order of factors in tensor product we obtain a natural isomorphism of $G$-modules

$$
\mathbf{V}(\Gamma) \simeq \bigotimes_{e \in E(\Gamma)}\left(V_{e} \otimes V_{e}^{*}\right)
$$

The space $\mathbf{V}(\Gamma)$ comes equipped with a symmetric bilinear form $\langle$,$\rangle defined on pure$ tensors $\mathbf{u}, \mathbf{w} \in \mathbf{V}$ by the following rule

$$
\langle\mathbf{u}, \mathbf{w}\rangle=\prod_{e \in E(\Gamma)} u_{e, t(e)}\left(w_{e, s(e)}\right) w_{e, t(e)}\left(u_{e, s(e)}\right),
$$

where

$$
\begin{aligned}
& \mathbf{u}=\bigotimes_{v \in V(\Gamma)}\left(\bigotimes_{e \in S(v)} u_{e, v}\right), \\
& \mathbf{w}=\bigotimes_{v \in V(\Gamma)}\left(\bigotimes_{e \in S(v)} w_{e, v)}\right) .
\end{aligned}
$$

Here $u_{e, s(e)}, w_{e, s(e)} \in V_{e}$ and $u_{e, t(e)}, w_{e, t(e)} \in V_{e}^{*}$

2.4.2. . It is clear that a principal $G$-bundle $E_{\Sigma}$ over $\Sigma$ defines a principal $G$-bundle $E_{\Gamma}$ over vertices of $\Gamma \subset \Sigma$. A connection $A$ on $E_{\Sigma}$ defines a graph connection on $E_{\Gamma}$ with the parallel transport along the edge $e$ being the parallel transport along $e \subset \Sigma$ with respect to connection $A$. The evaluation of the parallel transport along $e$ in the representation $V_{e}$ we will denote by $P_{e}^{A}: V_{e} \rightarrow V_{e}$.

Define the vector $\boldsymbol{\pi}^{A} \in \mathbf{V}$ in the $G$-module (10) as

$$
\pi^{A}=\bigotimes_{e \in E(\Gamma)} P_{e}^{A}
$$

Here we assume a choice of total ordering on the set of edges and the identification with the tensor product with the reordered tensor product as in (11). The holonomy map $P_{e}$ we regard as a vector in $V_{e} \otimes V_{e}^{*}$.

From the coloring of vertices we we get another vector $\mathbf{c}^{\Gamma} \in \mathbf{V}(\Gamma)$

$$
\mathbf{c}^{\Gamma}=\bigotimes_{v \in V(\Gamma)} c_{v}^{\Gamma}
$$

Here $c_{v}^{\Gamma}$ is the coloring of the vertex $v$ i.e. a vector in $V(v)^{G} \subset V(v)$.

Definition 10. Define graph functions using the symmetric bilinear form (12) as

$$
F_{\Gamma, V, c}(A)=\left\langle\pi^{A}, \mathbf{c}^{\Gamma}\right\rangle .
$$

Proposition 11. Graph functions have the following properties:

$$
F_{\Gamma, V, c}\left(A^{g}\right)=F_{\Gamma, V, c}(A)
$$

where $A^{g}$ is a flat connection A after gauge transformation $g$. If the connection is flat, the function $F_{\Gamma, V, c}(A)$ depends only on the isotopy class $[\Gamma]$ of $\Gamma$. 
Proof. By Definition $9, c_{p}^{\Gamma} \in V(p)^{G}$ is an invariant vector for each vertex $p$ of the graph. Hence, each function $F_{\Gamma, V, c}$ is invariant under gauge transformations of $A$. Moreover, when $A$ is flat connection, $F_{\Gamma_{1}, V, c}(A)=F_{\Gamma_{2}, V, c}(A)$ whenever an immersed graph $\Gamma_{1}$ can be deformed to $\Gamma_{2}$ by the regular homotopy (i.e. homotopy through graph immersions).

As a result, for each triple $([\Gamma], V, c)$ where $[\Gamma]$ is the isotopy class of an immersed oriented ordered graph $\Gamma, V$ is an edge coloring and $c$ is a vertex coloring we have a function $F_{[\Gamma], V, c}=F_{\Gamma, V, c}$ on the moduli space of flat connections on $\Sigma_{g, b}$. As a consequence, $F$ is a function of the moduli space of flat connections.

Theorem 12 [AMR96]. Functions $F_{[\Gamma], V, c}$ span the coordinate ring (7) of the moduli space of $G$-representations of the fundamental group $\pi_{1}\left(\Sigma_{g, b}, p\right)$.

Proof. By an algebraic analogue of Peter-Weyl theorem, the coordinate ring $R=$ $\mathcal{O}\left[G^{N}\right]$ of $G$-representations of the fundamental group can be decomposed as a $G^{N} \times$ $\left(G^{N}\right)^{o p}$-module

$$
\mathcal{O}\left[G^{N}\right] \simeq \bigoplus_{\lambda_{1}, \ldots, \lambda_{N} \in \Lambda}\left(\bigotimes_{j=1}^{N} W_{\lambda_{j}} \otimes W_{\lambda_{j}}^{*}\right)
$$

where $W_{\lambda}$ stands for a finite-dimensional irreducible representation with highest weight $\lambda$.

In particular, (15) is an isomorphism of $G$-modules, where $G$ acts by conjugation. Now let $Q \in \mathcal{O}\left[G^{N}\right]^{G}$ be a $G$-invariant polynomial, by (15) it can be decomposed as

$$
Q=\sum_{j=1}^{m} Q_{j}, \quad Q_{j} \in\left(W_{\lambda_{j, 1}} \otimes W_{\lambda_{j, 1}}^{*} \otimes \cdots \otimes W_{\lambda_{j, N}} \otimes W_{\lambda_{j, N}}^{*}\right)^{G}
$$

where each $Q_{j}$ is a graph function associated to the ribbon graph with a single vertex and $N=2 g+b-1$ morphisms corresponding to free generators of $\pi_{1}\left(\Sigma_{g, b}, p\right)$. The edge coloring of edges corresponding to generators of the fundamental group is given by $\lambda_{j, 1}, \ldots, \lambda_{j, N}$, while $Q_{j}$ is an invariant vector which defines the coloring of the single vertex $p$.

2.5. Equivalence of graph functions. In this subsection we list elementary operations on colored immersed graphs which leave the associated graph function (14) invariant. These operations include collapsing a pair of neighbouring vertices (Fig. 3c) and thinning a pair of neighbouring edges (Fig. 3d). In each case shown on Fig. 3 we refer to the left graph as $\Gamma_{1}$ and to the right graph as $\Gamma_{2}$.

Lemma 13 [FR99, KRWY]. Let $\Gamma_{1}, \Gamma_{2}$ be a pair of colored immersed oriented graphs which coincide away from a disc shown on any of Fig. $3 a-d$. Then there exists a vertex coloring of $\Gamma_{2}$, such that the two colored immersed graph give rise to the same invariant function on graph connections:

$$
F_{\left[\Gamma_{1}\right]}=F_{\left[\Gamma_{2}\right]} .
$$

Proof. Consider a flat connection $A$ on a principle $G$-bundle over $\Sigma_{g, b}$ and let

$$
\pi_{\Gamma_{1}}^{A}=\bigotimes_{e \in E\left(\Gamma_{1}\right)} P_{e} \in \mathbf{V}\left(\Gamma_{1}\right), \quad \pi_{\Gamma_{2}}^{A}=\bigotimes_{e \in E\left(\Gamma_{2}\right)} Q_{e} \in \mathbf{V}\left(\Gamma_{2}\right) .
$$




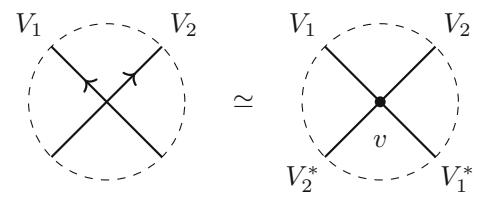

(A) Adding a vertex at transverse intersection

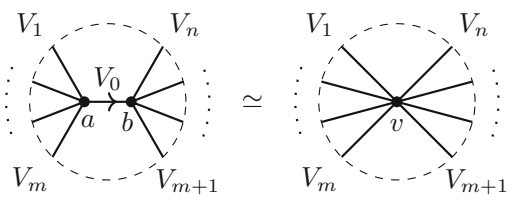

(C) Collapsing a pair of neighbouring vertices

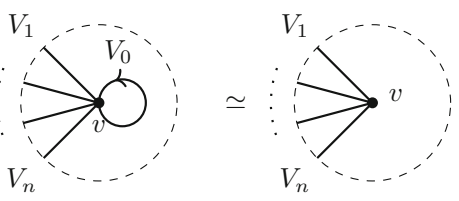

(B) Collapsing a contractible loop

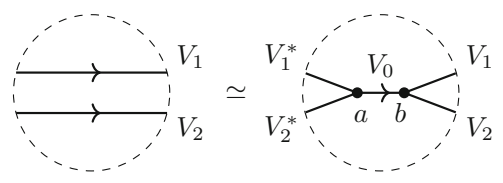

(D) Thinning a pair of neighbouring edges

Fig. 3. Graphs defining equivalent functions (Edges are oriented outwards unless specified)

be a pair of vectors in and as in (13) defined by the parallel transport along edges of the two immersed graphs. In each of the four parts we are going to prove that

$$
F_{\left[\Gamma_{1}\right]}(A)=\left\langle\pi_{\Gamma_{1}}^{A}, \mathbf{c}^{\Gamma_{1}}\right\rangle=\left\langle\pi_{\Gamma_{1}}^{A}, \mathbf{c}^{\Gamma_{2}}\right\rangle=F_{\left[\Gamma_{2}\right]}(A)
$$

for an appropriate choice of vertex coloring.

(A) Let

$$
\operatorname{Id}_{V_{1}}=\sum_{i} v_{i} \otimes v_{i}^{*}, \quad \operatorname{Id}_{V_{2}}=\sum_{j} w_{i} \otimes w_{j}^{*}
$$

where $\left\{v_{i}\right\},\left\{v_{i}^{*}\right\}$ and $\left\{w_{i}\right\},\left\{w_{i}^{*}\right\}$ be any two pairs of dual bases on $V_{1}, V_{1}^{*}$ and $V_{2}, V_{2}^{*}$ respectively, i.e. $\left\langle v_{i}, v_{j}^{*}\right\rangle=\delta_{i, j}$ and $\left\langle w_{i}, w_{j}^{*}\right\rangle=\delta_{i, j}$. Assign a vertex coloring of $\Gamma_{2}$ at $v$ as

$$
c_{v}^{\Gamma_{2}}=\sum_{i, j} v_{i} \otimes w_{j}^{*} \otimes v_{i}^{*} \otimes w_{j} \simeq \operatorname{Id}_{V_{1}} \otimes \operatorname{Id}_{V_{2}}
$$

(B) Let $\Gamma_{1}$ and $\Gamma_{2}$ be a pair of colored oriented immersed graphs which coincide away from a disc shown on Fig. 3b. Suppose that the vertex coloring of $\Gamma_{1}$ at $v$ is given by

$$
c_{v}^{\Gamma_{1}}=\sum_{j} v_{j}^{0} \otimes w_{j}^{(0)} \otimes v_{j}^{(1)} \otimes \cdots \otimes v_{j}^{(1)} .
$$

Define $c_{v}^{\Gamma_{2}}$ as follows

$$
c_{v}^{\Gamma_{1}}=\sum_{j}\left\langle v_{j}^{0}, w_{j}^{(0)}\right\rangle v_{j}^{(1)} \otimes \cdots \otimes v_{j}^{(1)}
$$


Because the holonomy of flat connection along contractible loop $e_{0}$ is trivial, we get the following contribution to holonomy vector (16)

$$
P_{e_{0}}=\operatorname{Id}_{V_{0}}=\sum_{i=1}^{M} u_{i} \otimes u_{i}^{*}
$$

where $\left\{u_{i}\right\}$ and $\left\{u_{i}^{*}\right\}$ is an arbitrary pair of dual bases in $V_{0}$ and $V_{0}^{*}$, i.e. $\left\langle u_{i}, u_{l}^{*}\right\rangle=\delta_{i, l}$. Then (17) follows immediately by

$$
\sum_{j=1}^{M}\left\langle w_{k}^{(0)}, u_{j}\right\rangle\left\langle u_{j}^{*}, v_{j}^{(0)}\right\rangle=\left\langle w_{k}^{(0)}, v_{j}^{(0)}\right\rangle
$$

(C) Assume further that the vertex coloring of vertices $a$ and $b$ is given by a pair of $G$-invariant vectors

$$
\begin{aligned}
& c_{a}^{\Gamma_{1}}=\sum_{j} v_{j}^{(0)} \otimes v_{j}^{(1)} \otimes \cdots \otimes v_{j}^{(m)} \in\left(V_{0} \otimes \cdots \otimes V_{m}\right)^{G}, \\
& c_{b}^{\Gamma_{1}}=\sum_{k} w_{k}^{(0)} \otimes w_{k}^{(m+1)} \otimes \cdots \otimes w_{k}^{(n)} \in\left(V_{0}^{*} \otimes V_{m+1} \otimes \cdots \otimes V_{n}\right)^{G} .
\end{aligned}
$$

Let $c_{v}$ be a $G$-invariant vector defined as follows:

$$
c_{v}^{\Gamma_{2}}=\sum_{j, k}\left\langle w_{k}^{(0)}, v_{j}^{(0)}\right\rangle v_{j}^{(1)} \otimes \cdots \otimes v_{j}^{(m)} \otimes w_{k}^{(m+1)} \otimes \cdots \otimes w_{k}^{(n)} \in\left(V_{1} \otimes \cdots \otimes V_{n}\right)^{G}
$$

Note that because both $F_{\left[\boldsymbol{\Gamma}_{1}\right]}$ and $F_{\left[\boldsymbol{\Gamma}_{2}\right]}$ are functions on gauge equivalence classes of flat connections, without loss of generality we can assume that holonomy of $A$ in a contractible disc on Fig. $3 c$ is trivial. Using the same reasoning as in (18) and (19) we conclude that (17) holds.

(D) For the remaining part, again without loss of generality we can assume that connection is trivial inside contractible disk shown on Fig. 3d. For $\varphi: V_{0} \stackrel{\sim}{\rightarrow} V_{1} \otimes V_{2}$, assign $G$-invariant vectors at both vertices induced by the isomorphism $\varphi$ and its inverse, the statement (17) then follows by tautology.

Corollary 14. Let $\Gamma \in \Sigma$ be an oriented immersed graph with a single vertex $p$ and edges corresponding to free generators of $\pi_{1}(\Sigma, p)$. For any oriented immersed graph $\left(\Gamma_{1}, W, d\right)$ we can present the associated graph function as

$$
F_{\left[\Gamma_{1}, W, d\right]}=F_{[\Gamma, V, c]}
$$

for an appropriate choice of coloring of simple oriented surface graph $\Gamma \subset \Sigma$.

Proof. Because $F_{\left[\Gamma_{1}, W, d\right]}$ depends only on homotopy (through immersed graphs) equivalence type of $\Gamma_{1}$, w.l.o.g. we can assume that $\Gamma \cup \Gamma_{1}$ has only double transverse intersection points. So by Lemma 13 we can turn $\Gamma \cup \Gamma_{1}$ first into an embedded graph, then collapse all vertices of $\Gamma_{1}$ into vertices of $\Gamma_{2}$ and, finally, by a sequence of moves Fig. 3b-d eliminate all vertices of $\Gamma_{1}$. 


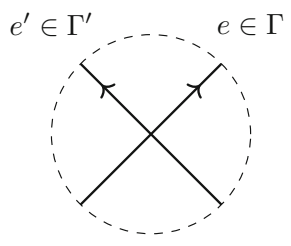

(A) Neighborhood of a simple intersection point $p \in \Gamma \cap \Gamma^{\prime}$

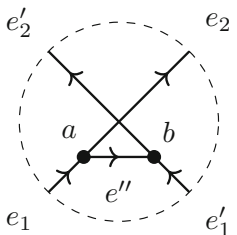

(B) The resulting immersed graph $\Gamma \star_{p} \Gamma^{\prime}$

Fig. 4. Double intersection point at edges and associated graph $\left(\Gamma, \Gamma^{\prime}\right)_{p}$

Replace holonomies along edges by groups elements $g_{e}$ given by a flat graph connection $g: E(\Gamma) \rightarrow G$ we identify the graph function $F_{[\Gamma, V, c]}$ with a function on the space flat graph connections for $\Gamma \subset \Sigma$. Taking into account isomorphisms (7) we can identify invariant graph functions with functions on the representation variety $\mathcal{M}_{\Sigma_{g, b}}^{G}$. Thus the corollary 14 establishes an isomorphism between the coordinate $\operatorname{ring} \mathcal{O}\left[\mathcal{M}_{\left(\Sigma_{g, b}, \Gamma\right)}\right]^{G}$ of moduli space of graph connections and the coordinte ring $\mathcal{O}\left[\mathcal{M}_{\Sigma_{g, b}^{G}}\right]^{G}$.

2.6. Poisson structure on the moduli space of flat connections. The coordinate ring $\mathcal{O}\left[\mathcal{M}_{\Sigma}^{G}\right]$ of the $G$-character variety of $\pi_{1}\left(\Sigma_{g, b}\right)$ comes equipped with a Poisson bracket equivariant under the action of the Mapping Class Group $\operatorname{Mod}\left(\Sigma_{g, b}\right)$ [AB83, Gol86, BG93]. Following [FR93,AMR96,FR99] we will define this bracket in terms of graph functions (14).

Let $([\Gamma], V, c)$ and $\left(\left[\Gamma^{\prime}\right], V^{\prime}, c^{\prime}\right)$ be a pair of isotopy equivalence classes of colored immersed graphs. Pick representatives $\Gamma$ and $\Gamma^{\prime}$ which have only transverse double intersection points at edges.

Definition 15. Let $p \in \Gamma \cap \Gamma^{\prime}$ be a transverse double intersection point of two colored immersed graphs $\boldsymbol{\Gamma}=(\Gamma, V, c)$ and $\Gamma^{\prime}=\left(\Gamma^{\prime}, V^{\prime}, c^{\prime}\right)$. Define (isotopy equivalence class of) colored immersed graph $\left[\Gamma \star_{p} \Gamma^{\prime}\right]=\left(\left[\Gamma^{\prime \prime}\right], V^{\prime \prime}, c^{\prime \prime}\right)$ as follows:

Consider a small neighborhood of $p$ and denote by $e \in \Gamma$ and $e^{\prime} \in \Gamma^{\prime}$ the corresponding edges of graphs containing the intersection point $p \in e \cap e^{\prime}$.

Take union of immersed graphs $\Gamma \cup \Gamma^{\prime}$ and add two extra vertices $a, b$ which divide edges $e, e^{\prime}$ into $e_{1}, e_{2}$ and $e_{1}^{\prime}, e_{2}^{\prime}$ respectively as shown on Fig. $4 \mathrm{~b}$. Then add an edge $e^{\prime \prime}$ connecting $a$ and $b$ colored by adjoint representation $V_{e^{\prime \prime}}^{\prime \prime}=\mathfrak{g}$ of $G .{ }^{11}$ Define vertex coloring

$$
\begin{aligned}
& c_{a}^{\prime \prime}=\left(\operatorname{ad}_{V_{e}} \otimes I d_{\mathfrak{g}}\right)(I) \quad \in \quad V_{e} \otimes V_{e}^{*} \otimes \mathfrak{g}, \\
& c_{b}^{\prime \prime}=\left(\operatorname{ad}_{V_{e^{\prime}}} \otimes I d_{\mathfrak{g}}\right)(I) \quad \in \quad V_{e^{\prime}} \otimes V_{e^{\prime}}^{*} \otimes \mathfrak{g},
\end{aligned}
$$

where $I=\sum_{i} t^{i} \otimes t_{i} \in \mathfrak{g} \otimes \mathfrak{g}$ is a quadratic Casimir. Finally, assume that edge and vertex coloring of the remaining graph is inherited from $\Gamma$ and $\Gamma^{\prime}$.

Theorem 16. [FR93,AMR96] The Poisson Bracket between $F_{[\mathbf{\Gamma}]}$ and $F_{\left[\boldsymbol{\Gamma}^{\prime}\right]}$ in terms of invariant graph functions reads

$$
\left\{F_{[\boldsymbol{\Gamma}]}, F_{\left[\boldsymbol{\Gamma}^{\prime}\right]}\right\}=\sum_{p \in \Gamma \cap \Gamma^{\prime}} \epsilon(p) F_{\left[\boldsymbol{\Gamma} \star_{p} \boldsymbol{\Gamma}^{\prime}\right]},
$$

\footnotetext{
11 Note that the adjoint representation is self-dual, so the orientation of $e^{\prime \prime}$ on Fig. $4 \mathrm{~b}$ can be chosen arbitrarily.
} 
where the sum is taken over the intersection points $p \in \Gamma \cap \Gamma^{\prime}$ and $\epsilon(p)= \pm 1$ stands for the sing of an intersection point.

\section{Main Construction}

The following theorem describes superintegrable systems on moduli spaces of flat connections. Poisson commuting Hamiltonians of these systems are invariant functions of holonomies along systems of nonintersecting simple curves on the surface.

Denote by $Z_{\partial \Sigma}$ the subalgebra of the coordinate ring $A=\mathcal{O}\left(\mathcal{M}_{\Sigma}^{G}\right)$ spanned by $G$ invariant functions on holonomies around boundary components. It must be a subalgebra of the Poisson center of $\mathcal{A}$. Moreover, it follows from [BG93] that $Z_{\partial \Sigma}$ has the same Krull dimension as the Poisson center of $A$.

Let $C=C_{1} \sqcup \ldots \sqcup C_{k}$ in $\Sigma$ be a disjoint union of pairwise nonhomotopic simple closed curves none of which is homopotic to a boundary component. Define $B_{C} \subset A$ as the subalgebra generated by graph functions $F_{\Gamma, V, c}$ with $\Gamma \subset C$. Similarly, define $J_{\Sigma \backslash C}$ as the subalgebra generated by graph functions $F_{\Gamma, V, c}$ with $\Gamma \subset(\Sigma \backslash C)$.

Theorem 17. The system of subalgebras below defines an affine superintegrable system

$$
Z_{\partial \Sigma} \subset B_{C} \subset J_{\Sigma \backslash C} \subset A=\mathcal{O}\left[\mathcal{M}_{\Sigma}^{G}\right]
$$

In the rest of this section we will prove this theorem. In order to prove it we should prove the balance of dimensions.

3.1. Trivial superintegrable system on a surface. Let $\Sigma$ be an oriented surface of genus $g$ with $b>0$ boundary components and let $\mathcal{M}_{\Sigma}^{G}$ be the moduli space of $G$-representations of the fundamental group $\pi_{1}(\Sigma)$. Assume that we have at least one boundary component. Denote by $Z_{\partial \Sigma} \subset A=\mathcal{O}\left[\mathcal{M}_{\Sigma}^{G}\right]$ a subalgebra generated by graph functions $F_{[\Gamma]}$, where $\boldsymbol{\Gamma}$ is a colored graph with a single vertex and a single edge homotopic to one of the boundary components of $\Sigma$.

Lemma 18. The subalgebra $Z_{\partial \Sigma} \subset \mathcal{O}\left[\mathcal{M}_{\Sigma}^{G}\right]$ belongs to the Poisson center of $\mathcal{O}\left[\mathcal{M}_{\Sigma}^{G}\right]$.

Proof. Let $\left[\boldsymbol{\Gamma}_{1}\right],\left[\boldsymbol{\Gamma}_{2}\right]$ be a pair of isotopy equivalence classes of colored immersed graphs such that $\left[\Gamma_{1}\right]$ is homotopic to one of the boundary components. Then there exist members $\boldsymbol{\Gamma}_{1}$ and $\boldsymbol{\Gamma}_{2}$ with a trivial intersection. From (21) we get

$$
\left\{F_{\left[\boldsymbol{\Gamma}_{1}\right]}, F_{\left[\boldsymbol{\Gamma}_{2}\right]}\right\}=0 .
$$

By Theorem 12, invariant graph functions of the form $F_{\left[\Gamma_{2}\right]}$ span the entire coordinate ring, hence we conclude that $F_{\left[\Gamma_{1}\right]}$ belongs to the Poisson center of $\mathcal{O}\left[\mathcal{M}_{\Sigma}^{G}\right]$.

Note that $Z_{\partial \Sigma}$ is generated by $b$ copies of $\mathcal{O}[G]^{G}$, one for each boundary component. In order to calculate Krull dimension of $Z_{\partial \Sigma}$ we will use the following pair of technical lemmas:

Lemma 19. Let $G$ be a connected simple linear algebraic group with a Lie Algebra $\mathfrak{g}$ and let

$$
\operatorname{ch}_{i} \in \mathcal{O}[G]^{G}, \quad 1 \leqslant i \leqslant \operatorname{rk}(G)
$$


be a maximal algebraically independent subset of $\mathcal{O}[G]^{G}$. Fix an arbitrary element $M_{0} \in G$, the following $3 \mathrm{rk}(G)$ functions on $G \times G$ are algebraically independent:

$$
G_{1, i}\left(M_{1}, M_{2}\right)=\operatorname{ch}_{i}\left(M_{1}\right), \quad G_{2, i}\left(M_{1}, M_{2}\right)=\operatorname{ch}_{i}\left(M_{2}\right), \quad G_{3, i}\left(M_{1}, M_{2}\right)=\operatorname{ch}_{i}\left(M_{0} M_{1} M_{2}\right),
$$

where $1 \leqslant i \leqslant \operatorname{rk}(G), M_{1}, M_{2} \in G$.

Proof. Note that collection of regular semisimple elements in $G$ forms an open dense subset, so generically both $M_{2}$ and $M_{2} M_{0} M_{1}$ are regular semisimple. Consider a pair of Cartan subgroups $\mathbf{T}_{M_{2}} \subset G$ and $\mathbf{T}_{M_{2} M_{0} M_{1}} \subset G$ containing $M_{2}$ and $M_{2} M_{0} M_{1}$ respectively. Denote by $\mathfrak{h}_{M_{2}}$ and $\mathfrak{h}_{M_{2} M_{0} M_{1}}$ their Cartan subalgebras. The two Cartan decompositions of $\mathfrak{g}$ acquire the following form

$$
\mathfrak{g}=\mathfrak{h}_{M_{2}} \oplus \mathfrak{h}_{M_{2}}^{\perp}=\mathfrak{h}_{M_{2} M_{0} M_{1}} \oplus \mathfrak{h}_{M_{2} M_{0} M_{1}}^{\perp}
$$

Now, for $X \in \mathfrak{g}$, consider a family of elements $\left(M_{1}, \mathrm{e}^{\epsilon X} M_{2} \mathrm{e}^{-\epsilon X}\right)$ on the common level set of $G_{1, i}$ and $G_{2, i}$. The remaining $\operatorname{rk}(G)$ class functions read

$$
\begin{aligned}
G_{3, i}\left(M_{1}, \mathrm{e}^{\epsilon X} M_{2} \mathrm{e}^{-\epsilon X}\right) & =\operatorname{ch}_{i}\left(M_{0} M_{1} \mathrm{e}^{\epsilon X} M_{2} \mathrm{e}^{-\epsilon X}\right) \\
& =\operatorname{ch}_{i}\left(M_{0} M_{1}\left(1+\epsilon\left(X-M_{2} X M_{2}^{-1}\right)\right) M_{2}\right)+\mathbf{o}(\epsilon) \\
& =\operatorname{ch}_{i}\left(M_{2} M_{0} M_{1}\left(1+\epsilon \pi_{\mathfrak{h}_{M_{2} M_{0} M_{1}}}\left(X-M_{2} X M_{2}^{-1}\right)\right)\right)+\mathbf{o}(\epsilon),
\end{aligned}
$$

where $\pi_{\mathfrak{h}_{M_{2} M_{0} M_{1}}}: \mathfrak{g} \rightarrow \mathfrak{h}_{M_{2} M_{0} M_{1}}$ stands for the projection map. Note that in the last equality we have used the fact that $\mathfrak{h}_{M_{2} M_{0} M_{1}}^{\perp}$-component of

$$
X-A d\left(M_{2}\right) X \quad \in \mathfrak{g}=\mathfrak{h}_{M_{2} M_{0} M_{1}} \oplus \mathfrak{h}_{M_{2} M_{0} M_{1}}^{\perp}
$$

in the first order in $\epsilon$ makes no contribution to the variation of $G_{3, i}$.

Recall that $M_{2}$ is regular semisimple, so dimension of its centralizer is exactly $\operatorname{rk}(G)$. As a corollary, the following endomorphism of the Lie algebra

$$
\Delta: \mathfrak{g} \rightarrow \mathfrak{g}, \quad \Delta(X)=X-A d_{M_{2}}(X)
$$

is nondegenerate on $\mathfrak{h}_{M_{2}}^{\perp}$. On the other hand, because $\mathfrak{h}_{M_{2}}$ and $\mathfrak{h}_{M_{2} M_{0} M_{1}}$ are in generic relative position to each other, this would imply that

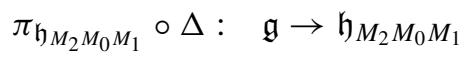

is surjective. Combining this fact with (24) we conclude the proof.

Lemma 20. As in the previous lemma, let $G$ be a connected simple linear algebraic group with a Lie Algebra $\mathfrak{g}$ and let

$$
\operatorname{ch}_{i} \in \mathcal{O}[G]^{G}, \quad 1 \leqslant i \leqslant \operatorname{rk}(G)
$$

be a maximal algebraically independent subset of $\mathcal{O}[G]^{G}$. Now fix an arbitrary element $M_{0} \in G$, the following $3 \operatorname{rk}(G)$ functions on $G \times G$ are algebraically independent:

$$
G_{1, i}\left(M_{1}, M_{2}\right)=\operatorname{ch}_{i}\left(M_{1}\right), \quad G_{2, i}\left(M_{1}, M_{2}\right)=\operatorname{ch}_{i}\left(M_{2}\right), \quad G_{3, i}\left(M_{1}, M_{2}\right)=\operatorname{ch}_{i}\left(M_{0}\left[M_{1}, M_{2}\right]\right),
$$

where $1 \leqslant i \leqslant \operatorname{rk}(G), M_{1}, M_{2} \in G$. 
Proof. Note that there exists an open dense subset $U \in G \times G$ such that for every $\left(M_{1}, M_{2}\right) \in U$ the following three elements

$$
\widetilde{M}_{0}=M_{2} M_{1}^{-1} M_{2}^{-1} M_{0} M_{1}, \quad \widetilde{M}_{1}=M_{2} M_{1}^{-1} M_{2}^{-1}, \quad M_{2}
$$

are regular semisimple. So without loss of generality we can assume $\left(M_{1}, M_{2}\right) \in U$ and also that $M_{1}$ and $M_{2}$ are in general relative position to each other. Denote by $\mathbf{T}_{\widetilde{M}_{0}}, \mathbf{T}_{\widetilde{M}_{1}}, \mathbf{T}_{M_{2}}$ their Cartan subgroups and by $\mathfrak{h}_{\widetilde{M}_{0}}, \mathfrak{h}_{\widetilde{M}_{1}}, \mathfrak{h}_{M_{2}}$ the corresponding Cartan subalgebras. We have

$$
\mathfrak{g}=\mathfrak{h}_{\widetilde{M}_{0}} \oplus \mathfrak{h} \frac{\perp}{\widetilde{M}_{0}}=\mathfrak{h}_{\widetilde{M}_{1}} \oplus \mathfrak{h} \frac{\perp}{\widetilde{M}_{1}}=\mathfrak{h}_{M_{2}} \oplus \mathfrak{h}_{M_{2}}^{\perp} .
$$

As in the previous lemma, for $\mathbf{X} \in \mathfrak{g}$, consider a family of elements $\left(M_{1}, \mathrm{e}^{\epsilon X} M_{2} \mathrm{e}^{-\epsilon X}\right)$ on the common level set of $G_{1, i}$ and $G_{2, i}$. For the remaining $\operatorname{rk}(G)$ class functions we get

$$
\begin{aligned}
& G_{3, i}\left(M_{1}, \mathrm{e}^{\epsilon X} M_{2} \mathrm{e}^{-\epsilon X}\right)=\operatorname{ch}_{i}\left(M_{0} M_{1} \mathrm{e}^{\epsilon X} M_{2} \mathrm{e}^{-\epsilon X} M_{1}^{-1} \mathrm{e}^{\epsilon X} M_{2}^{-1} \mathrm{e}^{-\epsilon X}\right) \\
& =\operatorname{ch}_{i}\left(M_{0} M_{1}\left(1+\epsilon\left(X-\operatorname{Ad}_{M_{2}} X+\operatorname{Ad}_{M_{2} M_{1}^{-1}} X-\operatorname{Ad}_{M_{2} M_{1}^{-1} M_{2}^{-1}} X\right)\right) M_{2} M_{1}^{-1} M_{2}^{-1}\right)+\mathbf{o}(\epsilon) \\
& =\operatorname{ch}_{i}\left(M_{2} M_{1}^{-1} M_{2}^{-1} M_{0} M_{1}\left(1+\epsilon\left(\operatorname{Id}-\operatorname{Ad}_{M_{2} M_{1}^{-1} M_{2}^{-1}}\right) \circ\left(\operatorname{Id}-\operatorname{Ad}_{M_{2}}\right)(X)\right)\right)+\mathbf{o}(\epsilon) \\
& =\operatorname{ch}_{i}\left(\widetilde{M}_{0}\left(1+\epsilon \pi_{\mathfrak{h}_{\widetilde{M_{0}}}} \circ\left(\operatorname{Id}-\operatorname{Ad}_{M_{2} M_{1}^{-1} M_{2}^{-1}}\right) \circ\left(\operatorname{Id}-\operatorname{Ad}_{M_{2}}\right)(X)\right)\right) \\
& =\operatorname{ch}_{i}\left(\widetilde{M}_{0}\left(1+\epsilon \pi_{\mathfrak{h}_{\mathbb{M}_{0}}} \circ\left(\operatorname{Id}-\operatorname{Ad}_{\widetilde{M}_{1}}\right) \circ \pi_{\mathfrak{h}_{\widetilde{M}_{1}}^{\perp}} \circ\left(\operatorname{Id}-\operatorname{Ad}_{M_{2}}\right)(X)\right)\right)
\end{aligned}
$$

Here in the last equality we have used the fact that $\operatorname{ker}\left(\operatorname{Id}-\operatorname{Ad}_{\widetilde{M}_{1}}\right)=\mathfrak{h}_{\widetilde{M}_{1}}$, so

$$
\operatorname{Id}-\operatorname{Ad}_{\tilde{M}_{1}}=\left(\operatorname{Id}-\operatorname{Ad}_{\tilde{M}_{1}}\right) \circ \pi_{\mathfrak{h}} \frac{1}{\tilde{M}_{1}} \cdot
$$

The statement of the Lemma will now follow from the fact that

$$
\pi_{\mathfrak{h}_{\widetilde{M}_{0}}} \circ\left(\operatorname{Id}-\operatorname{Ad}_{\widetilde{M}_{1}}\right) \circ \pi_{\mathfrak{h}} \frac{1}{\widetilde{M}_{1}} \circ\left(\operatorname{Id}-\operatorname{Ad}_{M_{2}}\right): \mathfrak{g} \rightarrow \mathfrak{h}_{\widetilde{M}_{0}}
$$

is surjective. To show that, recall that Id $-\operatorname{Ad}_{M_{2}}$ is nondegenerate on $\mathfrak{h}_{M_{2}}^{\perp}$. Because $\mathfrak{h}_{M_{2}}^{\perp}$ and $\mathfrak{h} \frac{\perp}{\widetilde{M}_{1}}$ are of the same dimension and are in general relative position, the restriction $\left.\pi_{\mathfrak{h}} \frac{1}{\widetilde{M}_{1}}\right|_{\mathfrak{h}} ^{\perp} \frac{\perp}{M_{2}}$ is a linear isomorphism between $\mathfrak{h}_{M_{2}}^{\perp}$ and $\mathfrak{h} \frac{\perp}{\widetilde{M}_{1}}$. Next, Id $-\operatorname{Ad}_{\widetilde{M}_{1}}$ is nondegenerate on $\mathfrak{h} \frac{\perp}{\widetilde{M}_{1}}$. Combining this with the fact that $\mathfrak{h}_{\widetilde{M}_{0}}$ and $\mathfrak{h} \frac{\perp}{\widetilde{M}_{1}}$ are in general relative position to each other we conclude the proof.

\section{Corollary 21.}

$$
\operatorname{dim} \operatorname{Spec} Z_{\partial \Sigma}= \begin{cases}(b-1) \operatorname{rk}(G), & g=0, b \leqslant 2, \\ b \operatorname{rk}(G), & \text { otherwise. }\end{cases}
$$

Proof. Let $p \in \partial \Sigma$ be a point of the boundary. With the choice of generators of $\pi_{1}(\Sigma, p)$ as in Sect. 2.1 we consider elements of $Z_{\partial \Sigma} \subset \mathcal{O}\left[\mathcal{M}_{\Sigma}^{G}\right]$ as $G$-invariant functions on $G^{\times(2 g+b-1)}$. In both cases the upper bound on dimension (27) follows by the fact that $Z_{\partial \Sigma}$ is generated by the appropriate number of copies of $\mathcal{O}[G]^{G}$, so the only thing we have to prove is that there exists a large enough algebraically independent subset of generators $Z_{\partial \Sigma}$.

Fix a maximal collection of algebraically independent class functions $\operatorname{ch}_{i} \in \mathcal{O}[G]^{G}$ and consider the following cases: 
(i) $b \geqslant 3$ : In this case $\left\{\operatorname{ch}_{i}\left(\rho\left(z_{j}\right)\right), 1 \leqslant i \leqslant \operatorname{rk}(G), 1 \leqslant j \leqslant b-3\right\}$ are algebraically independent as functions on $G^{\times(b-3)}$. At the same time, for fixed $\rho\left(z_{j}\right) \in G, 1 \leqslant$ $j \leqslant b-3$, the remaining $3 \operatorname{rk}(G)$ functions $\operatorname{ch}_{i}\left(\rho\left(z_{b-2}\right)\right), \operatorname{ch}_{i}\left(\rho\left(z_{b-1}\right)\right), \operatorname{ch}_{i}\left(\rho\left(z_{b}\right)\right)$ are independent as functions on the remaining $G \times G$ by Lemma 19. So, the entire collection of $3 \mathrm{rk}(G)$ functions must be algebraically independent.

(ii) $b=2, g>0$ : In this case we have a set of $2 \operatorname{rk}(G)$ algebraically independent functions $\operatorname{ch}_{i}\left(\rho\left(b_{1}\right)\right)$ and $\operatorname{ch}_{i}\left(\rho\left(b_{2}\right)\right)=\operatorname{ch}_{i}\left(\rho\left(b_{1}^{-1}\left[y_{g}, x_{g}\right] \ldots\left[y_{1}, x_{1}\right]\right)\right)$. To see it, recall that $\operatorname{ch}_{i}\left(\rho\left(b_{1}\right)\right)$ are algebraically idependent as functions on $G$ by construction. Next, apply Lemma 20 for

$$
M_{0}=\rho\left(b_{1}^{-1}\left[y_{g}, x_{g}\right] \ldots\left[y_{2}, x_{2}\right]\right), \quad M_{1}=y_{g}, \quad M_{2}=x_{g} .
$$

to conclude that $\operatorname{ch}_{i}\left(\rho\left(b_{1}^{-1}\left[y_{g}, x_{g}\right] \ldots\left[y_{1}, x_{1}\right]\right)\right)$ are algebraically idependent as functions on $G^{2 g}$, a fixed level set of $\rho\left(b_{1}\right)=$ const. Altogether this would imply that the entire collection of $2 \mathrm{rk}(G)$ functions are independent as functions on $G^{2 g+1}$.

(iii) $b=2, g=0$ : Here $\pi_{1}(\Sigma, p)=\left\langle b_{1}\right\rangle$ and we have $\operatorname{rk}(G)$ algebraically independent functions $\operatorname{ch}_{i}\left(\rho\left(b_{1}\right)\right)$.

(iv) $b=1, g>0$ : In this case we have $\operatorname{rk}(G)$ algebraically independent functions $\operatorname{ch}_{i}\left(\rho\left(\left[x_{1}, y_{1}\right] \ldots\left[x_{g}, y_{g}\right]\right)\right)$. Algebraic independence follows from Lemma 20 applied for

$$
M_{0}=\left[x_{1}, y_{2}\right] \ldots\left[x_{g-1}, y_{g-1}\right], \quad M_{1}=x_{g}, \quad M_{2}=y_{g} .
$$

(v) $b=1, g=0$ : Statement holds trivially.

Proposition 22. Inclusion $Z_{\partial \Sigma} \subset Z_{\partial \Sigma} \subset A \subset A$ defines an affine superintegrable system.

Proof. First, note that $Z_{\partial \Sigma}$ is finitely generated, indeed it is generated by $b$ copies of $\mathcal{O}[G]^{G}$, one for each boundary component. Every $\mathcal{O}[G]^{G}$ is in turn finitely generated.

By Lemma 18 we know that $Z_{\partial \Sigma}$ is a subalgebra of the Poisson center of $A$. On the other hand, by results of [GHJW97,BG93] we know that the general fiber of $\mathcal{M}_{\Sigma}^{G} \rightarrow$ $\operatorname{Spec} Z_{\partial \Sigma}$ is equipped with Poisson bracket of rank $\operatorname{dim} \mathcal{M}_{\Sigma}^{G}-\operatorname{dim} \operatorname{Spec} Z_{\partial \Sigma}$.

3.2. Superintegrable system associated to non-separating curve. Let $\Sigma$ be an oriented surface of genus $g$ with $b \geq 1$ boundary components. There exists no nonseparating curves on genus zero surface, so throught this section we can assume without loss of generality that $g>0$. Let $C=C_{1} \sqcup C_{2} \sqcup \ldots \sqcup C_{r}$ be a nonseparating curve which is a disjoint union of $r, 1 \leqslant r \leqslant g$ simple closed curves on $\Sigma$ (see Fig. 5).

The fundamental group $\pi_{1}(\Sigma, p)$ is a free group with $2 g+b-1$ generators, it admits the following presentation

$$
\pi_{1}(\Sigma, p)=\left\langle x_{1}, y_{1}, \ldots, x_{g}, y_{g}, z_{1}, \ldots, z_{b-1}\right\rangle
$$

where the first $2 r$ generators $x_{i}, y_{i}, \quad 1 \leqslant i \leqslant r$ are chosen for handles containing $C_{1}, \ldots, C_{r}$ as shown on Fig. 6. 


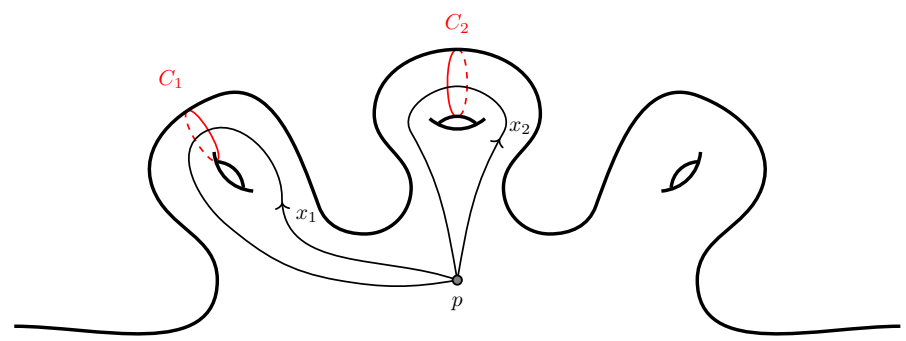

Fig. 5. An example of a nonseparating Curve

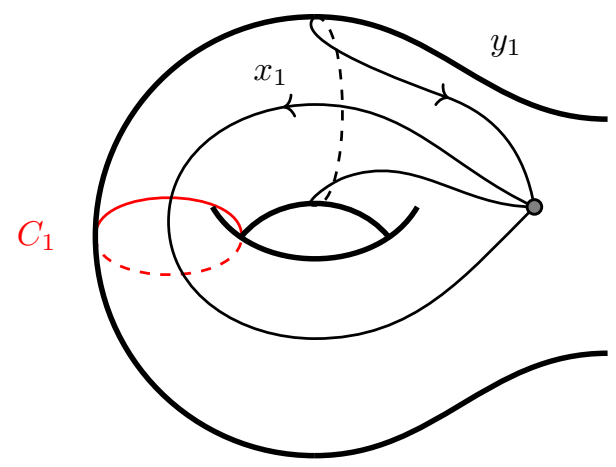

Fig. 6. Connected component of nonseparating curve

Lemma 23. Consider a subgroup of $\pi_{1}(\Sigma, p)$ which consists of (homotopy equivalence classes of) paths that do not cross $C$ :

$$
\pi_{1}(\Sigma, p \mid C) \subset \pi_{1}(\Sigma, p)
$$

This subgroup is generated by the following elements

$$
\pi_{1}(\Sigma, p \mid C)=\left\langle y_{1}, \ldots, y_{r}, x_{1} y_{1} x_{1}^{-1}, \ldots, x_{r} y_{r} x_{r}^{-1}, x_{r+1}, y_{r+1}, \ldots, x_{g}, y_{g}, z_{1}, \ldots, z_{b-1}\right\rangle .
$$

Proof. Note that there is a natural surjective homomorphism

$$
\psi: \quad \pi_{1}(\Sigma \backslash C, p) \rightarrow \pi_{1}(\Sigma, p \mid C)
$$

From the fundamental group of $\Sigma \backslash C$ to the subgroup (29). As a corollary, $\pi_{1}(\Sigma, p \mid C)$ is generated by the image of the generating set of $\pi_{1}(\Sigma \backslash C, p)$. Note that surface $\Sigma \backslash C$ has genus $g-r$ with $2 r+b$ boundary components. In the standard presentation, $\pi_{1}(\Sigma \backslash C, p)$ is generated by $(g-r)$ pairs of $A, B$-cycles along with $2 r+b-1$ boundary cycles. Taking the image of this generating set under $\psi$ we get (30).

The closed arcs homotopic to new boundary components are $y_{1}, \ldots, y_{r}$ and $x_{1} y_{1} x_{1}^{-1}$, $\ldots, x_{r} y_{r} x_{r}^{-1}$. As a result, $\pi_{1}(\Sigma \backslash C, p)$ is a free group generated by $2 g+b-1$ arcs.

A $G$-representation of $\pi_{1}(\Sigma, p)$ is determined by $2 g+b-1$ elements of $G$

$$
x_{i} \mapsto X_{i}, \quad y_{i} \mapsto Y_{i}, \quad z_{j} \mapsto Z_{j}, \quad 1 \leqslant i \leqslant g, \quad 1 \leqslant j \leqslant b-1 .
$$


As a corollary, $\operatorname{Hom}\left(\pi_{1}(\Sigma, p), G\right)$ is an affine variety with the coordinate ring $\mathcal{O}[$ Hom $\left.\left(\pi_{1}(\Sigma, p), G\right)\right] \simeq \mathcal{O}[G]^{\otimes(2 g+b-1)}$ isomorphic to the coordinate ring of $2 g+b-1$ copies of $G$.

Inclusion (29) defines an inclusion of coordinate rings

$$
\begin{array}{ccc}
\mathcal{O}\left[\operatorname{Hom}\left(\pi_{1}(\Sigma, p \mid C), G\right)\right] & \subset & \mathcal{O}\left[\operatorname{Hom}\left(\pi_{1}(\Sigma, p), G\right)\right] \\
\cup & & \cup \\
\mathcal{O}\left[\operatorname{Hom}\left(\pi_{1}(\Sigma, p \mid C), G\right)\right]^{G} & \subset & \mathcal{O}\left[\operatorname{Hom}\left(\pi_{1}(\Sigma, p), G\right)\right]^{G}
\end{array}
$$

At the same time, $\pi_{1}(\Sigma, p \mid C)$ contains $b$ infinite cyclic subgroups generated by boundary cycles. Let $Z_{\partial \Sigma}$ be a subalgebra of $\mathcal{O}\left[\mathcal{M}_{\Sigma}^{G}\right]$ generated by class functions of holonomies about the boundary components, or, equivalently, generated by $\mathcal{O}\left[\operatorname{Hom}\left(\left\langle z_{i}\right\rangle\right.\right.$, $G)]^{G}$ for $1 \leqslant i \leqslant b$.

Similarly, let $B_{C}$ be a subalgebra of $\mathcal{O}\left[\mathcal{M}_{\Sigma}^{G}\right]$ generated by $Z_{\partial \Sigma}$ together with traces of holonomies about the cycles $C_{i}$, in other words, by $\mathcal{O}\left[\operatorname{Hom}\left(\left\langle y_{i}\right\rangle, G\right)\right]^{G}$ for $1 \leqslant i \leqslant r$.

\section{Lemma 24.}

$$
\operatorname{dim} \operatorname{Spec} B_{C}=(b+r) \operatorname{rk}(G) .
$$

Proof. The upper bound on dimension follows from the fact that $B_{C}$ is generated by $b+r$ copies of $\mathcal{O}[G]^{G}$. Fix a maximal algebraically independent collection of class functions $\operatorname{ch}_{i} \in \mathcal{O}[G]^{G}$ and consider a family of $(b+r) \operatorname{rk}(G)$ elements of $B_{C}$ :

$$
\operatorname{ch}_{i}\left(\rho\left(y_{j}\right)\right), \quad \operatorname{ch}_{i}\left(\rho\left(z_{l}\right)\right), \quad 1 \leqslant i \leqslant \operatorname{rk}(G), \quad 1 \leqslant j \leqslant r, \quad 1 \leqslant l \leqslant b .
$$

Here we imply $z_{b}=z_{b-1}^{-1} \ldots z_{1}^{-1}\left[y_{g}, x_{g}\right] \ldots\left[y_{1}, x_{1}\right]$.

The first $(b+r-1) \operatorname{rk}(G)$ elements are algebraically independent as functions on $G^{2 g+b-1}$ because they involve different copies of $G$. As for the $\operatorname{ch}_{i}\left(\rho\left(z_{b}\right)\right)$, apply Lemma 20 for

$$
M_{0}=\rho\left(z_{b-1} \ldots z_{1}\left[y_{g}, x_{g}\right] \ldots\left[y_{2}, x_{2}\right]\right), \quad M_{1}=\rho\left(y_{1}\right), \quad M_{2}=\rho\left(x_{1}\right)
$$

to observe that restriction of $\operatorname{ch}_{i}\left(\rho\left(z_{b-1}^{-1} \ldots z_{1}^{-1}\left[y_{g}, x_{g}\right] \ldots\left[y_{1}, x_{1}\right]\right)\right)$ to generic common level set of the other $(b+r-1) \operatorname{rk}(G)$ elements gives rise to $\operatorname{rk}(G)$ independent functions.

Proposition 25. The following chain of subalgebras

$$
Z_{\partial \Sigma} \subset B_{C} \subset J_{\Sigma \backslash C} \subset \mathcal{O}\left[\mathcal{M}_{\Sigma}^{G}\right]
$$

defines an affine superintegrable system. Here $J=\mathcal{O}\left[\operatorname{Hom}\left(\pi_{1}(\Sigma, p \mid C), G\right)\right]^{G}$ stands for the coordinate ring of the $G$-character variety of $\pi_{1}(\Sigma, p \mid C)$.

Proof. By construction, all algebras involved are finitely generated and from (21) it follows immediately that $\{Z, A\}=\{B, J\}=0$. Hence $Z$ is a subalgebra of the Poisson center of $\mathcal{O}\left[\mathcal{M}_{\Sigma}^{G}\right]$ and $B$ is Poisson commutative and centralized by $J$.

Combining (33) with (27) we get

$$
\operatorname{dim} \operatorname{Spec} B_{C}-\operatorname{dim} \operatorname{Spec} Z_{\partial \Sigma}=r \operatorname{rk}(G) .
$$


On the other hand, any representation of $\pi_{1}(\Sigma, p \mid C)$ is determined by a $2 g+b-1$ elements of $G$

$$
Y_{i}, \quad \tilde{Y}_{j}, \quad X_{k}, \quad Z_{l}, \quad 1 \leqslant i \leqslant g, \quad 1 \leqslant j \leqslant r, \quad r+1 \leqslant k \leqslant g, \quad 1 \leqslant l \leqslant b-1,
$$

such that $\tilde{Y}_{j} \sim Y_{j}$ belong to the same conjugacy class, i.e. for all $1 \leqslant j \leqslant r$ we have $\tilde{Y}_{j}=X_{j} Y_{j} X_{j}^{-1}$ for some $X_{j}$. Note that for generic $Y_{j}$, dimension of its centralizer equals to $\operatorname{rk}(G)$. As a corollary, we get

$$
\operatorname{dim} \operatorname{Hom}\left(\pi_{1}(\Sigma, p \mid C), G\right)=(2 g+b-1) \operatorname{dim} G-r \operatorname{rk}(G) .
$$

Recall that for the purpose of this subsection we assume $g>0$ and $b>0$, so the action of $G$ by conjugation on $\operatorname{Hom}\left(\pi_{1}(\Sigma, p \mid C), G\right)$ has generically zero-dimensional stabilizer. As a corollary,

$$
\operatorname{dim} \operatorname{Spec} J_{\Sigma \backslash C}=(2 g+b-2) \operatorname{dim} G-r \operatorname{rk}(G) .
$$

Combining (8) with (35) we get

$$
\operatorname{dim} \mathcal{M}_{\Sigma}^{G}-\operatorname{dim} \operatorname{Spec} J_{\Sigma \backslash C}=r \operatorname{rk}(G) .
$$

Finally, comparing (34) with (36) we conclude that balance of dimensions (5) holds.

\subsection{Product of surface superintegrable systems associated with gluing.}

3.3.1. Choice of presentation of the fundamental groupoid Let $\Sigma$ be an oriented surface with $b>0$ boundary components and $C=C_{1} \sqcup \ldots \sqcup C_{r}$ be a disjoint union of $r$ simple closed curves in $\Sigma$ which cuts the surface into two parts $\Sigma=\Sigma_{L} \#_{C} \Sigma_{R}$. As in the statement of Theorem 1 we assume that none of the simple curves is homotopic to a boundary and all $C_{1}, \ldots, C_{r}$ are pairwise nonhomotopic. As a corollary, neither $\Sigma_{L}$, nor $\Sigma_{R}$ can be a cylinder. Further, because surface $\Sigma$ has nontrivial boundary $\partial \Sigma \neq \emptyset$, at least one of the surfaces $\Sigma_{L}, \Sigma_{R}$ has a boundary component not in $C$. Without loss of generality we can assume that $\partial \Sigma_{L} \supsetneq C$.

Because $\Sigma_{L}$ and $\Sigma_{R}$ both have at least one boundary component, their fundamental groups are free, say with $m$ and $n$ generators respectively. It will be convenient for us to choose the following presentation

$\pi_{1}\left(\Sigma_{L}, p\right)=\left\langle a_{1}, \ldots, a_{m}\right\rangle, \quad \pi_{1}\left(\Sigma_{R}, q\right)=\left\langle b_{1}, \ldots, b_{n}, b_{n+1} \mid b_{1}=F\left(b_{2}, \ldots, b_{n+1}\right)\right\rangle$.

Here $a_{1}, \ldots, a_{m}$ are free generators of $\pi_{1}\left(\Sigma_{L}\right)$. Because $\partial \Sigma_{L} \supsetneq C$, we can choose the first $r$ generators $a_{j}, \quad 1 \leqslant j \leqslant r$ to be homotopic to $C_{j}$ as shown on Fig. 7. At the same time, for $\Sigma_{R}$ we will choose the presentation as follows: Let $b_{2}, \ldots, b_{n+1}$ correspond to closed paths from $q$ to $q$ which freely generate $\pi_{1}\left(\Sigma_{R}, q\right)$. Without loss of generality we can assume that $b_{2}, \ldots, b_{r}$ are homotopic to $C_{2}, \ldots, C_{r}$. Now, let $b_{1}$ be the closed path homotopic to $C_{1}$, it can be expressed as a word in other generators $b_{1}=F\left(b_{2}, \ldots, b_{n+1}\right) .^{12}$

\footnotetext{
12 Adding extra generator $b_{1}$ for $\pi_{1}\left(\Sigma_{R}, q\right)$ satisfying relation $b_{1}=F\left(b_{2}, \ldots, b_{n+1}\right)$ allows us to treat both cases $\partial \Sigma_{R}=C$ and $\partial \Sigma_{R} \supsetneq C$ simultaneously without any changes in notation.
} 


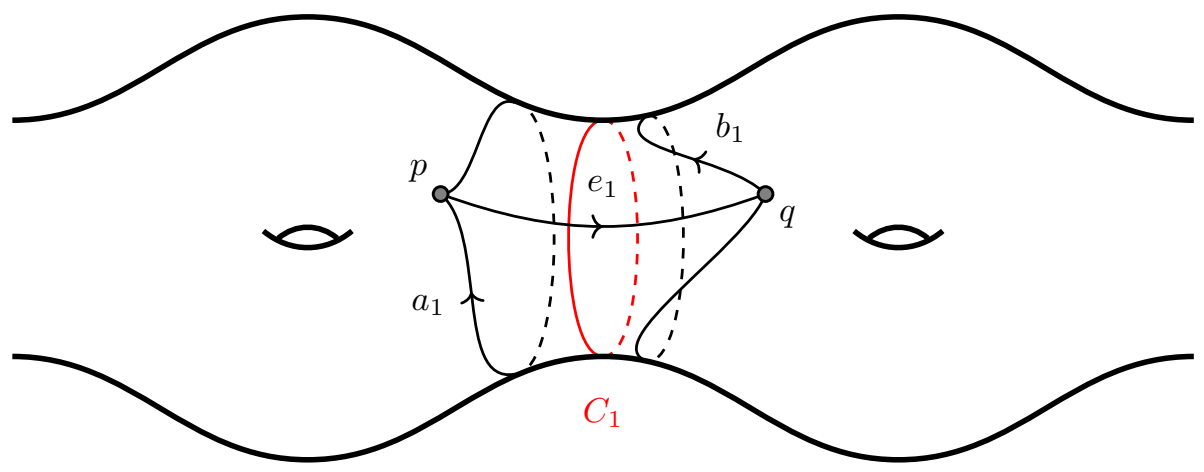

Fig. 7. Connected component of a separating curve

Denote by $\pi_{1}(\Sigma, p, q)$ the full subcategory of the fundamental groupoid $\pi_{1}(\Sigma)$ with two objects $p, q \in \Sigma .{ }^{13}$ By corollary of the Van Kampen's Theorem, groupoid $\pi_{1}(\Sigma, p, q)$ has the following presentation

$\pi_{1}(\Sigma, p, q)=\left\langle\begin{array}{l|l}a_{1}, \ldots, a_{m}, e_{1}, \ldots, e_{r}, b_{1}, \ldots, b_{n}, b_{n+1} \mid \begin{array}{l}a_{1}=e_{1}^{-1} b_{1} e_{1}, \ldots, a_{r}=e_{r}^{-1} b_{r} e_{r}, \\ b_{1}=F\left(b_{2}, \ldots, b_{n+1}\right)\end{array}\end{array}\right.$,

Here open arcs $e_{1}, \ldots, e_{r}$ stand for the paths connecting $p$ and $q$ which cross once exactly one of the separating cycles $C_{1}, \ldots, C_{r}$, see Figs. 7 and 8.

Note that $\pi_{1}(\Sigma, p, q)$ is freely generated by $a_{r+1}, \ldots, a_{m}, e_{1}, \ldots, e_{r}, b_{2}, \ldots, b_{n+1}$.

3.3.2. Coordinate rings of $G$-representations and relations between them Any $G$-representation of $\pi_{1}(\Sigma, p, q)$ is defined by $m+n+r+1$ elements subject to $r+1$ relations:

$$
\begin{aligned}
& A_{1}, \ldots, A_{m}, E_{1}, \ldots, E_{r}, B_{1}, \ldots, B_{n}, B_{n+1} \in G, \\
& B_{j} E_{j}=E_{j} A_{j}, \quad 1 \leqslant j \leqslant r, \quad B_{1}=F\left(B_{2}, \ldots, B_{n+1}\right) .
\end{aligned}
$$

This makes the set $\operatorname{Hom}\left(\pi_{1}(\Sigma, p, q), G\right)$ of all $G$-representations of $\pi_{1}(\Sigma, p, q)$ into an algebraic subset of $G^{\times(m+n+r+1)}$. Moreover, $\operatorname{Hom}\left(\pi_{1}(\Sigma, p, q), G\right)$ comes equipped with a $G \times G$ action:

$$
\begin{aligned}
A_{j} & \mapsto g A_{j} g^{-1}, & & 1 \leqslant j \leqslant m, \\
E_{k} \mapsto h E_{k} g^{-1}, & & 1 \leqslant k \leqslant r, & \\
B_{l} \mapsto h B_{l} h^{-1}, & & 1 \leqslant l \leqslant n+1, &
\end{aligned} \quad(g, h) \in G \times G,
$$

Of course, any $G$-representation of $\pi_{1}(\Sigma, p, q)$ also defines $G$-representations of $\pi_{1}\left(\Sigma_{L}, p\right)$ and $\pi_{1}\left(\Sigma_{R}, q\right)$. Explicitly, for our choice of generators we obtain a diagram of $G \times G$-equivariant projections

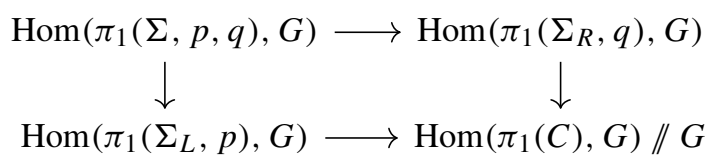

13 We use two distinct points $p \in \Sigma_{L}$ and $q \in \Sigma_{R}$ in order to simplify the comparison of the formulas with Sect. 4. 


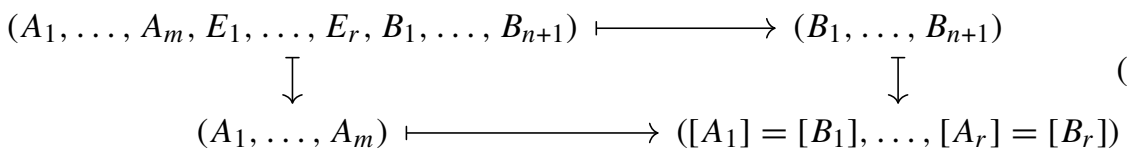

where $\left[A_{j}\right]=\left[B_{j}\right]$ stands for the common conjugacy class of $A_{j}$ and $B_{j}$.

Let $I$ be a $G \times G$-invariant ideal in $\mathcal{O}\left[G^{\times(m+n+r+1)}\right]$ defined by equations (39), then

$$
\mathcal{O}\left[\operatorname{Hom}\left(\pi_{1}(\Sigma, p, q), G\right)\right] \simeq \mathcal{O}\left[G^{\times(m+n+r+1)}\right] / I .
$$

In terms of the coordinate ring (42) of $G$-representations of $\pi_{1}(\Sigma, p, q)$ diagram (42) corresponds to the following diagram of inclusions of subalgebras

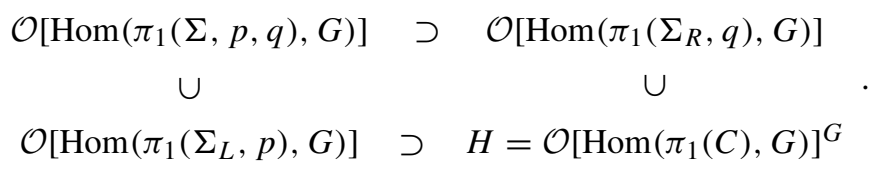

Here $H=\mathcal{O}\left[\operatorname{Hom}\left(\pi_{1}(C), G\right)\right]^{G}$ is the coordinate ring of a $G$-character variety of a disjoint union of $r$ circles corresponding to connected components of $C=\partial \Sigma_{L} \cap \partial \Sigma_{R}=$ $C_{1} \sqcup \ldots \sqcup C_{r}$. Hence $H \simeq\left(\mathcal{O}[G]^{G}\right)^{\otimes r}$ is generated by invariant functions on common conjugacy classes $\left[A_{i}\right]=\left[B_{i}\right], 1 \leqslant i \leqslant r$ of matrices $A$ and $B$.

\section{Lemma 26.}

$$
H=\mathcal{O}\left[\operatorname{Hom}\left(\pi_{1}\left(\Sigma_{L}, p\right)\right)\right] \cap \mathcal{O}\left[\operatorname{Hom}\left(\pi_{1}\left(\Sigma_{R}, q\right)\right)\right]
$$

Proof. From (43) we already know that $H \subset \mathcal{O}\left[\operatorname{Hom}\left(\pi_{1}\left(\Sigma_{L}, p\right)\right)\right] \cap \mathcal{O}\left[\operatorname{Hom}\left(\pi_{1}\left(\Sigma_{R}, q\right)\right)\right]$, so we have to prove only the opposite inclusion. To this end, first recall that all algebras in (43) are $G \times G$-modules w.r.t. the action (40). Moreover, $1 \times G$ is acting trivially on $\mathcal{O}\left[\operatorname{Hom}\left(\pi_{1}\left(\Sigma_{L}, p\right)\right)\right]$, while $G \times 1$ is acting trivially on $\mathcal{O}\left[\operatorname{Hom}\left(\pi_{1}\left(\Sigma_{R}, q\right)\right)\right]$. As a corollary, their intersection lies in the $G$-invariant part of both subalgebras $\mathcal{O}\left[\operatorname{Hom}\left(\pi_{1}\right.\right.$ $\left.\left.\left(\Sigma_{L}, p\right)\right)\right] \cap \mathcal{O}\left[\operatorname{Hom}\left(\pi_{1}\left(\Sigma_{R}, q\right)\right)\right] \subset \mathcal{O}\left[\mathcal{M}_{\Sigma_{L}}^{G}\right] \cap \mathcal{O}\left[\mathcal{M}_{\Sigma_{R}}^{G}\right]$. By Theorem 12 combined with Corollary 14 we conclude that the right hand side of (44) is spanned by invariant graph functions on $C$, hence contained in $H$.

Now consider the following tensor product over $H$

$$
J_{0}=\mathcal{O}\left[\operatorname{Hom}\left(\pi_{1}\left(\Sigma_{L}, p\right), G\right)\right] \underset{H}{\otimes} \mathcal{O}\left[\operatorname{Hom}\left(\pi_{1}\left(\Sigma_{R}, q\right), G\right)\right]
$$

By Lemma 26, $J \subset \mathcal{O}\left[\operatorname{Hom}\left(\pi_{1}(\Sigma, p, q), G\right)\right]$, this gives us another subalgebra of $\mathcal{O}\left[\operatorname{Hom}\left(\pi_{1}(\Sigma, p, q), G\right)\right]$ which fits in the middle of diagram (43).

By construction, all rings involved in (43) and (45) are finitely generated integral domains. Hence, taking the spectrum of all rings in (43) and (45) we obtain a commutative diagram of $G \times G$-equivariant dominant maps of affine schemes 


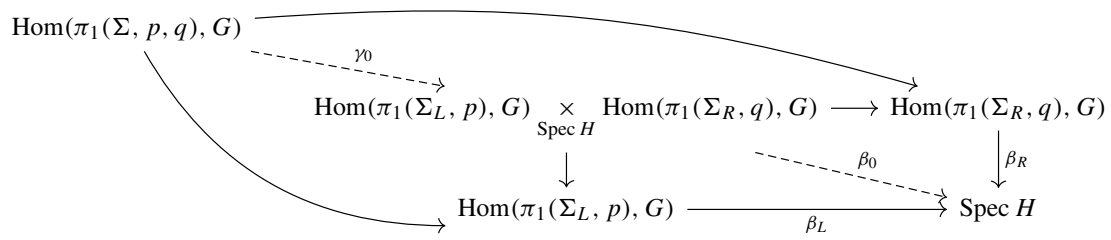

where $\operatorname{Spec}(H) \simeq(G / / \operatorname{Ad} G)^{r}$ and

$$
\operatorname{Hom}\left(\pi_{1}\left(\Sigma_{L}, p\right), G\right) \underset{\operatorname{Spec} H}{\times} \operatorname{Hom}\left(\pi_{1}\left(\Sigma_{R}, q\right), G\right)=\operatorname{Spec} J_{0}
$$

is the fiber product of maps $\beta_{L}$ and $\beta_{R}$.

3.3.3. Product of superintegrable systems Taking the GIT quotient by $G \times G$ action of diagram (46) we obtain from the main diagonal the following chain of dominant maps of affine schemes

$$
\begin{aligned}
& \operatorname{Hom}\left(\pi_{1}(\Sigma, p, q), G\right) / /(G \times G) \stackrel{\gamma}{\rightarrow}\left(\operatorname{Hom}\left(\pi_{1}\left(\Sigma_{L}, p\right), G\right) \underset{\operatorname{Spec} H}{\times} \operatorname{Hom}\left(\pi_{1}\left(\Sigma_{R}, q\right), G\right)\right) \\
& / /(G \times G) \stackrel{\beta}{\longrightarrow} \operatorname{Spec} H
\end{aligned}
$$

Note that by (7), we have natural isomorphism

$$
\operatorname{Hom}\left(\pi_{1}(\Sigma, p, q), G\right) / /(G \times G) \simeq \mathcal{M}_{\Sigma}^{G}
$$

Remark 27. Key idea of the remaining part of this subsection is that projections (47) define an affine superintegrable system on $\mathcal{O}\left[\mathcal{M}_{\Sigma}^{G}\right]$ associated with the separating collection of cycles $C=\partial \Sigma_{L} \cap \partial \Sigma_{R}=C_{1} \sqcup \ldots \sqcup C_{r}$.

Moreover, we can actually prove a much more general statement, formulated in Proposition 29, that if each of the surfaces $\Sigma_{L}, \Sigma_{R}$ was already equipped with an affine superintegrable system on $\mathcal{O}\left[\mathcal{M}_{\Sigma_{L}}\right]$ and $\mathcal{O}\left[\mathcal{M}_{\Sigma_{R}}\right]$ respectively, then gluing along $C$ provides a way to construct a new superintegrable system on $\Sigma$ which refines (47).

Lemma 28. Equation (47) defines a chain of Poisson projections.

Proof. Note that the neighbourhood of $C$ is a disjoint union of $r$ annuli. Recall that $H \simeq\left(\mathcal{O}[G]^{G}\right)^{\otimes r}$ is the tensor product of $r$ copies of the coordinate ring of the moduli space of $G$-representations of an annulus. It is naturally equipped with the trivial Poisson structure and with two natural injective Poisson homomorphisms

$$
H \stackrel{\beta_{L}^{*}}{\longrightarrow} \mathcal{O}\left[\mathcal{M}_{\Sigma_{L}}^{G}\right] \quad H \stackrel{\beta_{R}^{*}}{\longrightarrow} \mathcal{O}\left[\mathcal{M}_{\Sigma_{R}}^{G}\right]
$$

to the Poisson center of the coordinate rings of $G$-character varieties of $\pi_{1}\left(\Sigma_{L}, p\right)$ and $\pi_{1}\left(\Sigma_{R}, q\right)$ respectively. As a result,

$$
H \stackrel{\beta^{*}}{\longrightarrow} J=\mathcal{O}\left[\mathcal{M}_{\Sigma_{L}}^{G}\right] \underset{H}{\otimes} \mathcal{O}\left[\mathcal{M}_{\Sigma_{R}}^{G}\right]
$$


is a Poisson embedding into the Poisson center of $J$. To finalize the proof, note that

$$
J \hookrightarrow \gamma^{*} \operatorname{Hom}\left(\pi_{1}(\Sigma, p, q), G\right)^{G \times G} \simeq \mathcal{O}\left[\mathcal{M}_{\Sigma}^{G}\right]
$$

is a subalgebra of the full coordinate ring of the moduli space of $G$-representations of $\pi_{1}(\Sigma, p, q)$.

Proposition 29. Suppose that we have a pair of affine superintegrable systems on $\Sigma_{L}$ and $\Sigma_{R}$ defined by some choice of nonseparating cycles $C_{L} \subset \Sigma_{L}$ and $C_{R} \subset \Sigma_{R}$ :

$$
Z_{L} \subset B_{L} \subset J_{L} \subset A_{L}=\mathcal{O}\left[\mathcal{M}_{\Sigma_{L}}^{G}\right], \quad Z_{R} \subset B_{R} \subset J_{R} \subset A_{R}=\mathcal{O}\left[\mathcal{M}_{\Sigma_{R}}^{G}\right],
$$

where $Z_{L}$ and $Z_{R}$ denote Poisson centers of $A_{L}$ and $A_{R}$ respectively. Then

$$
Z \subset B \subset J \subset A=\mathcal{O}\left[\mathcal{M}_{\Sigma}^{G}\right]
$$

defines an affine superintegrable system on $\Sigma$. Here $Z$ is the Poisson center of $\mathcal{O}\left[\mathcal{M}_{\Sigma}^{G}\right]$,

$$
B=B_{L} \underset{H}{\otimes} B_{R}, \quad J=J_{L}{\underset{H}{\otimes}}_{R} J_{R},
$$

and $H=\mathcal{O}\left[\mathcal{M}_{C}^{G}\right]$ is the coordinate ring of the $G$-character variety of $\pi_{1}(C) \simeq$ Free ${ }_{r}$. Proof. First, note that

$$
\left\{\mathcal{O}\left[\mathcal{M}_{\Sigma_{L}}^{G}\right], \mathcal{O}\left[\mathcal{M}_{\Sigma_{R}}^{G}\right]\right\}=0
$$

where $\{$,$\} stands for the Poisson bracket on \mathcal{M}_{\Sigma}^{G}$. Indeed, by Proposition 12 subalgebras $\mathcal{O}\left[\mathcal{M}_{\Sigma_{L}}^{G}\right]$ and $\mathcal{O}\left[\mathcal{M}_{\Sigma_{R}}^{G}\right]$ are spanned by invariant graph functions which have no intersections. Combining it with the fact that $\left\{B_{L}, J_{L}\right\}=0=\left\{B_{R}, J_{R}\right\}$ we conclude that $\{B, J\}=0$, and, in particular, that $B$ has a trivial Poisson bracket. So the only thing we have to prove is that Krull dimensions of the algebras satisfy (5).

Krull dimensions of algebras $B$ and $J$ are determined as follows ${ }^{14}$

$\operatorname{dim} B=\operatorname{dim} B_{L}+\operatorname{dim} B_{R}-\operatorname{dim} H, \quad \operatorname{dim} J=\operatorname{dim} J_{L}+\operatorname{dim} J_{R}-\operatorname{dim} H$.

Now denote by $g_{L}$ and $b_{L}$ the genus and number of boundary components of $\Sigma_{L}$. Similarly, let $g_{R}$ and $b_{R}$ denote the genus and number of boundary components of $\Sigma_{R}$. The resulting surface $\Sigma$ then has a genus $g=g_{L}+g_{R}+(r-1)$ and number of boundary components $b=b_{L}+b_{R}-2 r$. Note that we have assume that neither $\Sigma_{L}$ nor $\Sigma_{R}$ is a cylinder (i.e. $\left.\left(g_{L}, b_{L}\right) \neq(0,2) \neq\left(g_{R}, b_{R}\right)\right)$. From (52) we have

$$
\operatorname{dim} B+\operatorname{dim} J=\operatorname{dim} B_{L}+\operatorname{dim} B_{R}+\operatorname{dim} J_{L}+\operatorname{dim} J_{R}-2 \operatorname{dim} H
$$

On the other hand, because both systems (49) are superintegrable, by (5) we conclude that

$$
\operatorname{dim} B+\operatorname{dim} J=\operatorname{dim} A_{L}+\operatorname{dim} Z_{L}+\operatorname{dim} A_{R}+\operatorname{dim} Z_{R}-2 \operatorname{dim} H .
$$

\footnotetext{
14 These two formulas is an algebraic version of a statement that for a pair of projections of irreducible affine varieties $X \rightarrow Z$ and $Y \rightarrow Z$ over $\mathbb{C}$, the dimension of the corresponding fiber product is $\operatorname{dim}\left(X \times{ }_{Z} Y\right)=$ $\operatorname{dim} X+\operatorname{dim} Y-\operatorname{dim} Z$.

Note that in order for (52) to be valid it is crucial that all algebras involved are finitely generated over $\mathbb{C}$ and have no zero divisors. Identities (52) then follow from the fact that transcendence degree of a field extension is additive w.r.t. the composition of field extensions.
} 
Finally, using explicit formula (8) for Krull dimensions of $A_{L}, A_{R}$ and (27) for $Z_{L}, Z_{R}$ we obtain

$$
\begin{aligned}
\operatorname{dim} B+\operatorname{dim} J & =\left(\left(2 g_{L}+b_{L}-2\right)+\left(2 g_{R}+b_{R}-2\right)\right) \operatorname{dim} G+\left(b_{L}+b_{R}-2 r\right) \operatorname{rank}(G) \\
& =(2 g+b-2) \operatorname{dim} G+b \operatorname{rank}(G) \\
& =\operatorname{dim} A+\operatorname{dim} Z .
\end{aligned}
$$

Hence, condition (5) on Krull dimensions of subalgebras (50) is satisfied and the resulting system is superintegrable.

\subsection{The proof of Theorem 17. Now we can prove our main result.}

Proof. Let $\Sigma \backslash C=\Sigma_{1} \sqcup \ldots \sqcup \Sigma_{m}$, where $\Sigma_{1}, \ldots, \Sigma_{m}$ stand for connected components. We will prove the statement of the theorem by induction in the number $m$ of connected components.

The base case $m=1$ follows by Proposition 25 .

Now assume that the statement of the theorem holds for all $\left(\Sigma^{\prime}, C^{\prime}\right)$ s.t. $\Sigma^{\prime} \backslash C^{\prime}$ has at most $m$ connected components. Then for each pair $(\Sigma, C)$ s.t. $\Sigma \backslash C$ has $m+1$ connected components we can present $\Sigma=\Sigma_{L} \sqcup \Sigma_{R}$, where both $\Sigma_{L} \backslash C$ and $\Sigma_{R} \backslash C$ have at most $m$ connected components. Define

$$
C_{L}=C \backslash \partial \Sigma_{R}, \quad C_{R}=C \backslash \partial \Sigma_{L}, \quad C_{L R}=\partial \Sigma_{L} \cap \partial \Sigma_{R}
$$

Note that $C=C_{L} \sqcup C_{L R} \sqcup C_{R}$. By the inductive assumption we have two superintegrable systems

$$
Z_{\partial \Sigma_{L}} \subset B_{C_{L}} \subset J_{\Sigma \backslash C_{L}} \subset \mathcal{O}\left[\mathcal{M}_{\Sigma_{L}}^{G}\right], \quad Z_{\partial \Sigma_{R}} \subset B_{C_{R}} \subset J_{\Sigma \backslash C_{R}} \subset \mathcal{O}\left[\mathcal{M}_{\Sigma_{R}}^{G}\right] .
$$

Now, applying Proposition 29 we finalize the proof.

The following proposition is an immediate consequence.

Proposition 30. Let $\Sigma$ be an oriented closed surface with $b>0$ boundary components and $C=C_{1} \sqcup \ldots \sqcup C_{k}$ and $C^{\prime}=C_{1}^{\prime} \sqcup \ldots \sqcup C_{m}^{\prime}$ be two collections of simple closed curves none of which is homotopic to a boundary component. Denote by $\mathcal{I}_{C}$ and $\mathcal{I}_{C^{\prime}}$ the two superintegrable systems associated to $C$ and $C^{\prime}$ as in Theorem 17.

- If $C \subset C^{\prime}$ is a subcollection of $C^{\prime}$, the integrable system $\mathcal{I}_{C}$ is a refinement of $\mathcal{I}_{C^{\prime}}$.

- If an element of the Mapping Class Group of surface $\Sigma$ bring the collection $C$ to $C^{\prime}$, it induces an equivalence of corresponding suerintegrable systems.

Remark 31. It is natural to expect that for an affine superintegrable system

$$
Z_{\partial \Sigma} \subset B_{C} \subset J_{\partial \Sigma \backslash C} \subset \mathcal{O}\left[\mathcal{M}_{\Sigma}^{G}\right]
$$

subalgebra $Z_{\partial \Sigma}$ is the full Poisson center of $\mathcal{O}\left[\mathcal{M}_{\Sigma}^{G}\right]$, and this is true in the compact case. Note that this implies that $J_{\Sigma \backslash C}$ is the full Poisson centralizer of $B_{C}$. 
3.5. Beyond generic orbits. The main result of the paper, formulated in Theorem 1 , states that a collection of simple closed curves $C=C_{1} \sqcup \ldots \sqcup C_{r}$ on an oriented surface $\Sigma$ defines a family of superintegrable systems. This family is parameterized by generic values of Casimir functions, in other words, by fixing holonomies around boundary components at regular conjugation orbits. We have left details of the case of nongeneric values of Casimir functions outside of the scope of the current paper.

We expect to prove the following stronger statement in a sequel publication. Consider a superintegrable system from Theorem 1 and let

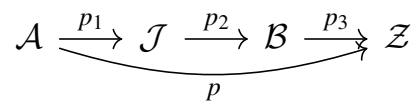

be an associated chain of dominant maps of affine schemes preserving the Poisson bracket. Then for all $z \in \operatorname{Spec} \mathcal{Z}$, every irreducible component of the fiber $p^{-1}(z)$ contains an open subset $\mathcal{M}_{2 n}$ such that

$$
\mathcal{M}_{2 n} \stackrel{\pi_{1}}{\longrightarrow} \mathcal{P}_{2 n-k} \stackrel{\pi_{2}}{\longrightarrow} \mathcal{B}_{k}
$$

is a superintegrable system. Here $\pi_{1}, \pi_{2}$ are maps induced by $p_{1}$ and $p_{2}$ respectively.

Example of systems with lower rank orbits were studied in [CF18]. In that case $G=$ $S L_{m}$, the surface $\Sigma$ is a torus with $n$ punctures, the conjugacy class of the monodromy around each puncture is fixed and is assumed to be of rank 1 . It is easy to see that the dimension of the moduli space in this case is $2(m-1) n$. The choice of $C=C_{1}, \ldots C_{n}$ being separating cycles cutting the torus into $n$ cylinders with one puncture in each cylinder produces a Liouville integrable system with $(m-1) n$ Poisson commuting integrals. For any $C_{i}$, the choice $C=C_{i}$ gives a superintegrable refinement of this Liouville integrable system.

\section{Hamilton Flows on the Full Coordinate Ring}

The Poisson bracket $(21)$ on the coordinate ring $\mathcal{O}\left[\operatorname{Hom}\left(\pi_{1}\left(\Sigma, p_{1}, \ldots, p_{m}\right), G\right)\right]^{G}$ of the moduli space $\mathcal{M}_{\Sigma}^{G}$ can be obtained from the bivector field on $R=\mathcal{O}\left[\operatorname{Hom}\left(\pi_{1}(\Sigma\right.\right.$, $\left.\left.\left.p_{1}, \ldots, p_{m}\right), G\right)\right]$ using two essentially different methods. One way is the " $r$-matrix approach" developed in [FR93], the other way is the "Quasi Poisson" bracket approach developed in [AMM98, AK00, AKM02].

In the $r$-matrix approach one defines Poisson bracket on the full coordinate ring

$$
\{,\}_{r-\text { matrix }}: R \otimes R \rightarrow R, \quad R=\mathcal{O}\left[\operatorname{Hom}\left(\pi_{1}\left(\Sigma, p_{1}, \ldots, p_{m}\right), G\right)\right]
$$

which coincides with the Goldman bracket on the $G$-invariant part $R^{G} \simeq \mathcal{O}\left[\mathcal{M}_{\Sigma}^{G}\right]$.

In the Quasi Poisson approach the bivector field on $\operatorname{Hom}\left(\pi_{1}\left(\Sigma, p_{1}, \ldots, p_{m}\right), G\right)$ is not Poisson. The bracket $\{,\}_{\text {quasi }}: R \otimes R \rightarrow R$ satisfies the Jacobi identity only up to a fixed trivector field vanishing on $R^{G}$. As in the $r$-matrix approach, quasi Poisson bracket induces the Goldman bracket on $R^{G}$

$$
\left.\{,\}_{\text {r-matrix }}\right|_{R^{G} \otimes R^{G}}=\left.\{,\}_{\text {quasi }}\right|_{R^{G} \otimes R^{G}}=\{,\}_{\text {Goldman }}: \quad R^{G} \otimes R^{G} \rightarrow R^{G} .
$$


Here we will focus on the $r$-matrix approach. ${ }^{15}$ The bracket (53) makes $R$ into a left Lie module over $R^{G}$. In other words, the $G$-invariant part acts on the full coordinate ring by derivations

$$
\{,\}: \quad R^{G} \otimes R \rightarrow R
$$

satisfying

$$
\begin{aligned}
& \{h, a b\}=\{h, a\} b+a\{h, b\}, \quad \text { for all } h \in R^{G}, \quad a, b \in R, \\
& \left\{h_{1},\left\{h_{2}, a\right\}\right\}-\left\{h_{2},\left\{h_{1}, a\right\}\right\}=\left\{\left\{h_{1}, h_{2}\right\}_{\text {Goldman }}, a\right\}, \quad \text { for all } h_{1}, h_{2} \in R^{G}, \quad a \in R .
\end{aligned}
$$

Action (54) allows one to integrate Hamilton flows described in Theorem 17. Moreover, with the appropriate choice of generators of the fundamental groupoid, this action acquires a remarkably simple form presented in Theorem 34 below. First, in in Sect. 4.1, for a given collection $C$ of pairwise nonintersecting simple closed curves we define a particular choice of marked points and generating arcs of $\pi_{1}\left(\Sigma, p_{1}, \ldots, p_{m}\right)$. The proposed choice of generating arcs allows us to simultaneously separate variables for Hamilton flows corresponding to different curves in $C$. Next, in Sect. 4.2 we use r-matrix formalism to calculate the Hamiltonian flow (54) associated to a simple closed curve in $C$ colored by a class function.

4.1. Choice of generators of $\pi_{1}$. Let $\Sigma$ be, as before, an oriented surface with nonempty boundary $\partial \Sigma=D_{1} \sqcup \ldots \sqcup D_{b}$ and let $C=C_{1} \sqcup \ldots \sqcup C_{k}$ be a disjoint collection of simple closed curves as in Theorem 17. Namely, we require that $C_{j}$ are pairwise nonintersecting, pairwise nonhomotopic, and none of them are homotopic to the boundary. Let $\Sigma \backslash C=\Sigma_{1} \sqcup \ldots \sqcup \Sigma_{m}$, where $\Sigma_{1}, \ldots, \Sigma_{m}$ stand for connected components. For the purpose of this section we further assume that $\partial \Sigma_{j} \cap \partial \Sigma \neq \emptyset$ for all $j \in\{1, \ldots, m\}$. This assumption can be made with no loss generality when we describe Hamilton flows, because initial conditions with trivial monodromy about the boundary component effectively correspond to absence of the boundary component.

For any connected component $\Sigma_{j}$ we choose a single marked point $p_{j} \in \partial \Sigma_{j} \cap \partial \Sigma$ on the common boundary with $\Sigma$. Each surface $\Sigma_{j}$ is an oriented surface of genus $g_{j}$ with $b_{j}$ boundary components. Its boundary can be presented as a disjoint union

$$
\partial \Sigma_{j}=D_{\beta_{j, 1}} \sqcup \ldots \sqcup D_{\beta_{j, r_{j}}} \sqcup C_{\alpha_{j, r_{j}+1}} \sqcup \ldots \sqcup C_{\alpha_{j, b_{j}}}
$$

of $r_{j}$ connected components $D_{\beta_{j, 1}}, \ldots, D_{\beta_{j, r_{j}}} \subset \partial \Sigma$ of the boundary of the full surface $\Sigma$, as well as $b_{j}-r_{j}$ connected components $C_{\alpha_{j, r_{j}+1}}, \ldots, C_{\alpha_{j, b_{j}}} \subset C$ of a curve $C$. Without loss of generality we can assume that $p_{j} \in D_{\beta_{j, 1}}$.

Denote by

$$
H_{j}=\left(C \cap \Sigma_{j}\right) \backslash \partial \Sigma_{j}=C_{\gamma_{j, 1}} \sqcup \ldots \sqcup C_{\gamma_{j, s_{j}}},
$$

the subcollection of simple closed curves in $C$ which belong to $\Sigma_{j}$, but do not belong to $\partial \Sigma_{j}$. Such subcollection is nonseparating by definition.

We will choose a set of generators of $\pi_{1}\left(\Sigma, p_{1}, \ldots, p_{n}\right)$ as follows:

\footnotetext{
15 For the analogue of the current section in terms of quasi Poisson bracket see [AR].
} 


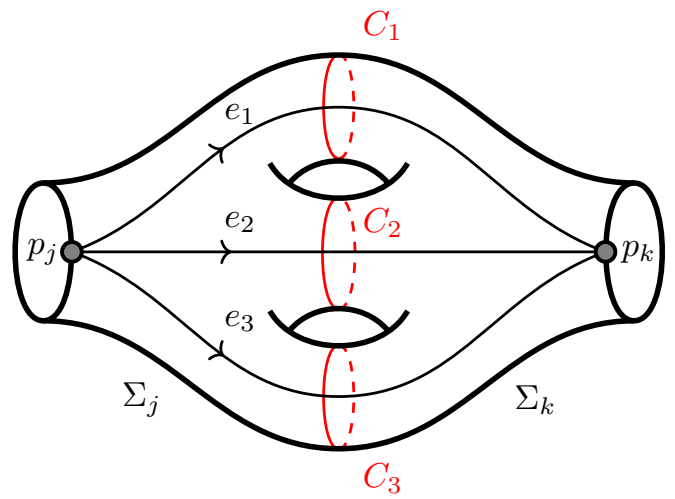

Fig. 8. Adjacent pair $\Sigma_{j} \cup \Sigma_{k}$

(1) First, for each simple closed curve $C_{i} \in \partial \Sigma_{j} \cap \partial \Sigma_{k}$ which separates a pair of distinct connected components $\Sigma_{j} \neq \Sigma_{k}$ of $\Sigma \backslash C$ we choose a simple arc $e_{i} \in$ $\left(\Sigma_{j} \backslash H_{j}\right) \cup\left(\Sigma_{k} \backslash H_{k}\right)$ from $p_{j}$ to $p_{k}$ passing once through $C_{j}$ as shown on Fig. 8 .

Note that without loss of generality we can assume that different $e_{j}$ intersect only at the endpoints. This can be achieved by sliding all intersection points between $e_{i}$ and $e_{i^{\prime}}$ in $\Sigma_{j}$ to $p_{j}$ followed by left multiplication by an element of $\pi_{1}\left(\Sigma_{j}, p_{j}\right)$. Similarly, one can get rid of the intersection points in $\Sigma_{k}$.

(2) For each arc $e_{i}$ chosen on the previous step we pick a simple closed curve $a_{i}$ which starts and ends at $p_{j}$ and homotopic to $C_{i}$ as shown on Fig. 7.

Again, we can assume that none of the $a_{i}, e_{l}$ intersect away from the endpoints. This can be achieved by choosing $a_{i}$ sufficiently close to $e_{i} \cup C_{i}$. Also, each $a_{i}$ chosen on this step cuts $\Sigma_{j}$ into two connected components, one of them is a cylinder given by the neighbourhood of $C_{i}$, the other is equivalent to the original surface. In what follows we assume that all further curves never enter into the cylinder.

(3) For each nonseparating simple closed curve $C_{\gamma_{j, k}}$ from (55) we choose a pair of simple closed curves $x_{\gamma_{j, k}}$ and $y_{\gamma_{j, k}}$, where $y_{\gamma_{j, k}}$ is homotopic to $C_{\gamma_{j, k}}$, while $x_{\gamma_{j, k}}$ passes once through $C_{\gamma_{j, k}}$ as shown on Fig. 6.

(4) At the last step we consider $\Sigma_{j} \backslash C$. This surface has genus $g_{j}-s_{j}$ and $b_{j}+2 s_{j}$ boundary components. Our choice of arcs already generates the paths around $r_{j}+2 s_{j}$ boundary components of $\Sigma_{j} \backslash C$. We can choose the remaining $2 g_{j}+b_{j}-r_{j}$ free generators of $\pi_{1}\left(\Sigma_{j} \backslash C\right)$ arbitrarily.

Collection of marked points and free generators of $\pi_{1}\left(\Sigma, p_{1}, \ldots, p_{m}\right)$ chosen above defines an ordered oriented ribbon graph with vertices $p_{1}, \ldots, p_{m}$ and edges corresponding to generators. The total order of half-edges adjacent to the given vertex is defined by an orientation of the surface. An example of the total order of half-edges is given on Fig. 9.

Data of a ribbon graph can be encoded in $m$ ordered sets of half-edges adjacent to $p_{j}$

$$
S_{j}=\left\{h_{j, 1}, \ldots, h_{j, n_{j}}\right\}, \quad 1 \leqslant j \leqslant m .
$$

Our ribbon graph is oriented, hence it will be convenient for us to label half-edges by generators of $\pi_{1}\left(\Sigma, p_{1}, \ldots, p_{m}\right)$ and its inverses. We associate a generator for an outgoing edge and inverse of a generator for an ingoing edge. For example, ordered set 


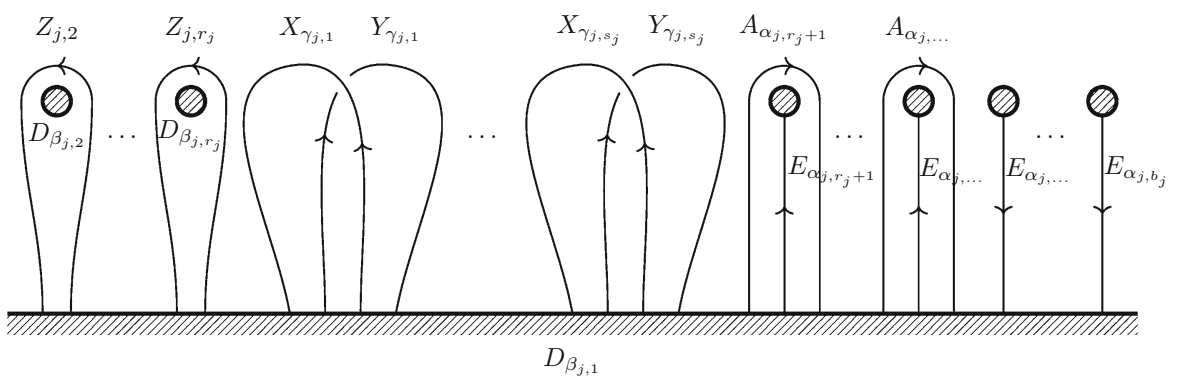

Fig. 9. An example of a total order of half-edges adjacent to $p_{j} \in \partial \Sigma_{j}$ (left to right)

of half-edges adjacent to $p_{j}$ on Fig. 9 reads

$$
S_{j}=\left\{z_{j, 2}^{-1}, z_{j, 2}, \ldots, x_{\gamma_{j, 1}}^{-1}, y_{\gamma_{j, 1}}, x_{\gamma_{j, 1}}, y_{\gamma_{j, 1}}^{-1}, \ldots, a_{\alpha_{j, r_{j}+1}}, e_{\alpha_{j, r_{j}+1}}, a_{\alpha_{j, r_{j}+1}}^{-1}, \ldots, e_{\alpha_{j}, b_{j}}^{-1}\right\} .
$$

4.2. The r-matrix approach. Let $\mathfrak{g}$ be a Lie algebra of $G$ and $r \in \mathfrak{g} \otimes \mathfrak{g}$ be a classical r-matrix, i.e. a a solution to the Yang-Baxter equation

$$
\left[r_{12}, r_{13}\right]+\left[r_{12}, r_{23}\right]+\left[r_{13}, r_{23}\right]=0
$$

We will be interested in solutions corresponding to factorizable Lie bialgebras, i.e. classical $r$-matrices with the following symmetric part (with respect to the exchange of tensor components) given by the Killing form:

$$
I=r+\sigma(r)=\sum_{J=1}^{\operatorname{dim} \mathfrak{g}} e_{J} \otimes e_{J}
$$

Here $\left\{e_{J}\right\}$ is a basis in $\mathfrak{g}$ which is orthonormal with respect to the Killing form and $\sigma(x \otimes y)=y \otimes x$. Such solutions are classified in [BD82].

The choice of free generators of $\pi_{1}\left(\Sigma, p_{1}, \ldots, p_{m}\right)$ fixes a natural isomorphism $\operatorname{Hom}\left(\pi_{1}\left(\Sigma, p_{1}, \ldots, p_{m}\right), G\right) \simeq G^{N}$ where $N=2 g+b+m-2$. For an ordered ribbon graph Fock and Rosly [FR93] introduced a Poisson structure on this space with the Poisson tensor defined in terms of factorizable classical $r$-matrices. Below we present the corresponding Poisson brackets between matrix element functions.

For two matrix valued functions $M_{i j}$ and $N_{a b}$ on a Poisson manifold we will use the following notation

$$
\{M \otimes N\}_{(i a)(j b)}=\left\{M_{i j}, N_{a b}\right\}
$$

for the matrix of Poisson brackets. Here the convention is $(M \otimes N)_{(i a)(j b)}=M_{i j} N_{a b}$.

Distinct arcs Basic building block of brackets corresponds to the case shown on Fig. 10a when the two oriented arcs share the source but have distinct targets different from their common source. 


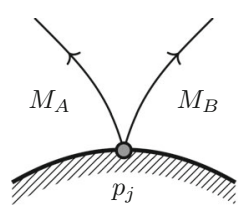

(A) Outgoing-Outgoing

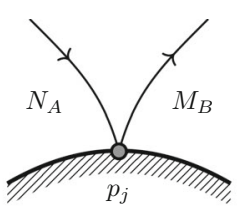

(B) Ingoing-Outgoing

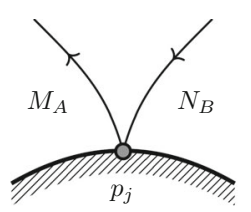

(c) Outgoing-Ingoing

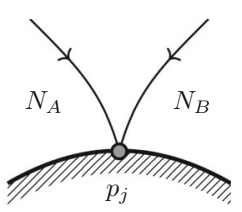

(D) Ingoing-Ingoing

Fig. 10. Relative position of half edges

In this case the Fock and Rosly Poisson bracket between matrix elements ${ }^{16}$ of holonomies along these arcs reads

$$
\left\{M_{A} \otimes M_{B}\right\}=\left(M_{A} \otimes M_{B}\right) r .
$$

One can easily calculate the Poisson bracket between matrix elements of powers and products of matrices. For example, by Leibnitz identity applied to $M_{A} M_{A}^{-1}=\mathrm{Id}=$ $M_{B} M_{B}^{-1}$ from (58a) we immediately obtain Poisson brackets for other three cases shown on Fig. 10b-d

$$
\begin{aligned}
& \left\{N_{A} \otimes M_{B}\right\}=-\left(\mathrm{Id} \otimes M_{B}\right) r\left(N_{A} \otimes \mathrm{Id}\right), \\
& \left\{M_{A} \otimes N_{B}\right\}=-\left(M_{A} \otimes \mathrm{Id}\right) r\left(\mathrm{Id} \otimes N_{B}\right), \\
& \left\{N_{A} \otimes N_{B}\right\}=r\left(N_{A} \otimes N_{B}\right),
\end{aligned}
$$

where $N_{A}=M_{A}^{-1}$ and $N_{B}=M_{B}^{-1}$.

Similarly, brackets $\left\{M_{B} \otimes M_{A}\right\}, \ldots,\left\{N_{B} \otimes N_{A}\right\}$ can be obtained by skew-symmetry, and the answer can be expressed in terms of $r_{21}$ as follows

$$
\begin{aligned}
& \left\{M_{B} \otimes M_{A}\right\}=-\left(M_{B} \otimes M_{A}\right) r_{21}, \\
& \left\{M_{B} \otimes N_{A}\right\}=\left(M_{B} \otimes \mathrm{Id}\right) r_{21}\left(\mathrm{Id} \otimes N_{A}\right), \\
& \left\{N_{B} \otimes M_{A}\right\}=\left(\mathrm{Id} \otimes M_{A}\right) r_{21}\left(N_{B} \otimes \mathrm{Id}\right), \\
& \left\{N_{B} \otimes N_{A}\right\}=-r_{21}\left(N_{B} \otimes N_{A}\right) .
\end{aligned}
$$

Here $r_{21}=\sigma(r)$.

Bracket between matrix elements of a general distinct pair of arcs now can be computed by simply adding up to four terms of the form (58) and (59) corresponding to brackets between pairs of half edges whenever they are adjacent to the same vertex.

Self-brackets There are only three cases of relative position of half edges of the same arc as shown on Fig. 11.

Corresponding brackets are given by the following formulae

$\{E \otimes E\}=r_{a}(E \otimes E)+(E \otimes E) r_{a}$,

$\left\{A_{R} \otimes A_{R}\right\}=\left(A_{R} \otimes A_{R}\right) r_{a}+r_{a}\left(A_{R} \otimes A_{R}\right)+\left(\mathrm{Id} \otimes A_{R}\right) r_{21}\left(A_{R} \otimes \mathrm{Id}\right)-\left(A_{R} \otimes \mathrm{Id}\right) r_{12}\left(\mathrm{Id} \otimes A_{R}\right)$,

$\left\{A_{L} \otimes A_{L}\right\}=r_{a}\left(A_{L} \otimes A_{L}\right)+\left(A_{L} \otimes A_{L}\right) r_{a}+\left(A_{L} \otimes \mathrm{Id}\right) r_{21}\left(\mathrm{Id} \otimes A_{L}\right)-\left(\mathrm{Id} \otimes A_{L}\right) r_{12}\left(A_{L} \otimes \mathrm{Id}\right)$.

where $r_{a}=\frac{1}{2}\left(r_{12}-r_{21}\right)$ is the antisymmetric (with respect to the exchange of tensor components) part of r-matrix and we write $r_{12}$ for $r$.

\footnotetext{
16 Here and below by matrix elements we always assume matrix elements in a finite dimensional representation.
} 

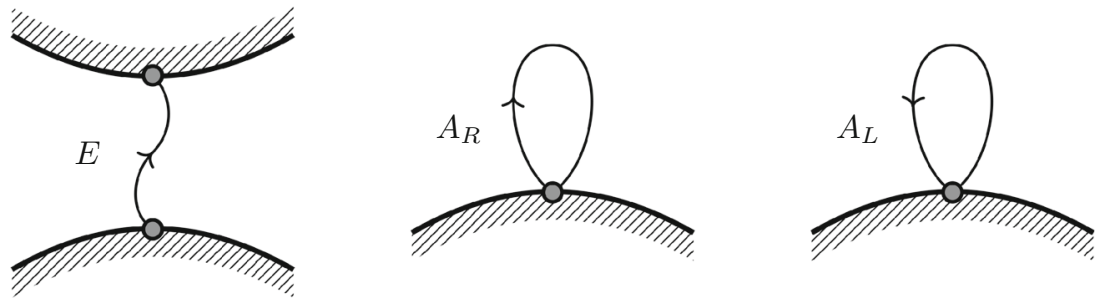

Fig. 11. Relative poisition of half edges of the same arc

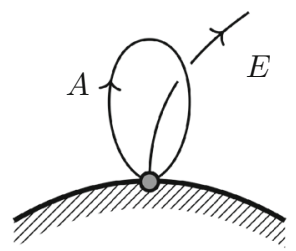

(A) Separating pair

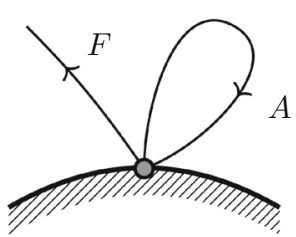

(в) Disjoint left

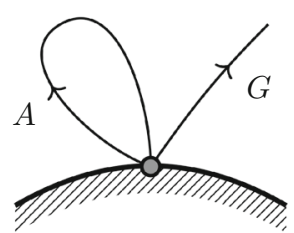

(C) Disjoint right

Fig. 12. Relative order of half-edges w.r.t to the closed arc

4.3. Hamiltonian flows generated by central functions. In this section we compute the evolution of matrix element functions on $\operatorname{Hom}\left(\pi_{1}\left(\Sigma, p_{1}, \ldots, p_{m}\right), G\right)$ with respect to the Hamiltonian flows generated by central functions on holonomies around simple closed $\operatorname{arcs} a_{i}$ or $y_{j}$ defined in Sect. 4.1. We start by considering three basic cases shown on Fig. 12.

Proposition 32. Let $(a, e),(f, a),(a, g)$ be pairs of generators with relative order of half-edges as shown on Fig. 12, $H: G \rightarrow \mathbb{C}$ be a polynomial central function, $H\left(g h g^{-1}\right)=H(h)$ and $\pi: G \rightarrow E n d(V)$ be a finite dimensional representation of $G$. Denote by $A, E, F, G \in \operatorname{Hom}\left(\pi_{1}\left(\Sigma, p_{1}, \ldots, p_{m}\right), G\right)$ holonomies along the corresponding oriented arcs. We have

$$
\begin{aligned}
& \left\{H(A), \pi_{V}(E)\right\}=\sum_{J}\left(e_{J}, \nabla H(A)\right) \pi_{V}\left(E e_{J}\right), \\
& \left\{H(A), \pi_{V}(F)\right\}=\left\{H(A), \pi_{V}(G)\right\}=\left\{H(A), \pi_{V}(A)\right\}=0 .
\end{aligned}
$$

where $e_{J}$ is a basis in $\mathfrak{g}$ orthonormal with respect to the Killing form and

$$
(\nabla H(A), X)=\left.\frac{d}{d t} H\left(e^{t X} A\right)\right|_{t=0}
$$

Here $X \in \mathfrak{g}$ and $(.,$.$) is the Killing form.$

Proof. It is enough to consider $H(A)=\operatorname{Tr} A^{k}$ where trace is taken over a finite dimensional representation (which can be different from $V$ ). Poisson bracket between matrix elements of $A$ and $E$ can be calculated as a sum of two terms: first term of the form (58a) corresponds to the ordered pair of half edges $(a, e)$ adjacent to the same vertex; second term of the form (59c) corresponds to the ordered pair of half edges $\left(e, a^{-1}\right)$. As a result, we have

$$
\{A \otimes E\}=(A \otimes E) r_{12}+(\mathrm{Id} \otimes E) r_{21}(A \otimes \mathrm{Id})
$$


Hence, by Leibnitz Identity for all $k \in \mathbb{N}$ we get

$$
\begin{aligned}
\left\{A^{k} \otimes E\right\} & =\sum_{j=0}^{k-1}\left(A^{j} \otimes \mathrm{Id}\right)\{A \otimes E\}\left(A^{k-j-1} \otimes \mathrm{Id}\right) \\
& =\sum_{j=0}^{k-1}\left(\left(A^{j+1} \otimes E\right) r_{12}\left(A^{k-j-1} \otimes \mathrm{Id}\right)+\left(A^{j} \otimes E\right) r_{21}\left(A^{k-j} \otimes \mathrm{Id}\right)\right) .
\end{aligned}
$$

Taking partial trace with respect to the first component we obtain (61a) as

$$
\begin{aligned}
\left\{\operatorname{Tr} A^{k}, E\right\} & =\operatorname{Tr}_{1}\left\{A^{k} \otimes E\right\} \\
& =\sum_{j=0}^{k-1} \operatorname{Tr}_{1}\left(\left(A^{j+1} \otimes E\right) r_{12}\left(A^{k-j-1} \otimes \mathrm{Id}\right)\right)+\sum_{j=0}^{k-1} \operatorname{Tr}_{1}\left(\left(A^{j} \otimes E\right) r_{21}\left(A^{k-j} \otimes \mathrm{Id}\right)\right) \\
& =\sum_{j=0}^{k-1} \operatorname{Tr}_{1}\left(\left(A^{k} \otimes E\right) r_{12}\right)+\sum_{j=0}^{k-1} \operatorname{Tr}_{1}\left(\left(A^{k} \otimes E\right) r_{21}\right)=k \operatorname{Tr}_{1}\left(\left(A^{k} \otimes E\right) I\right) .
\end{aligned}
$$

Poisson bracket between matrix elements of $A$ and $G$ can be calculated as a sum of two terms: first term of the form (58a) corresponds to ordered pair of half edges $(a, g)$; second term of the form (58b) corresponds to ordered pair of half edges $\left(a^{-1}, g\right)$. Hence, we have

$$
\{A \otimes G\}=(A \otimes G) r-(\mathrm{Id} \otimes G) r(A \otimes \mathrm{Id}) .
$$

By Leibnitz Identity we get that for all $k \in \mathbb{N}$

$$
\left\{A^{k} \otimes G\right\}=\sum_{j=0}^{k-1}\left(\left(A^{j+1} \otimes G\right) r\left(A^{k-j-1} \otimes \mathrm{Id}\right)-\left(A^{j} \otimes G\right) r\left(A^{k-j} \otimes \mathrm{Id}\right)\right) .
$$

Taking partial trace with respect to the first component we get

$$
\begin{aligned}
\left\{\operatorname{Tr} A^{k}, G\right\} & =\sum_{j=0}^{k-1} \operatorname{Tr}_{1}\left(\left(A^{j+1} \otimes G\right) r\left(A^{k-j-1} \otimes \mathrm{Id}\right)\right)-\sum_{j=0}^{k-1} \operatorname{Tr}_{1}\left(\left(A^{j} \otimes G\right) r\left(A^{k-j} \otimes \mathrm{Id}\right)\right) \\
& =k \operatorname{Tr}_{1}\left(\left(A^{k} \otimes G\right) r\right)-k \operatorname{Tr}_{1}\left(\left(A^{k} \otimes G\right) r\right)=0 .
\end{aligned}
$$

Poisson bracket between matrix elements of $A$ and $F$ is determined by (59a) and (59c) and has the following form

$$
\{A \otimes F\}=-(A \otimes F) r_{21}+(\mathrm{Id} \otimes F) r_{21}(A \otimes \mathrm{Id})
$$

Note that $r_{21}$ is on the same side from $F$ in both terms of (62), so as in the previous case we obtain for all $k \in \mathbb{N}$

$$
\left\{\operatorname{Tr} A^{k}, F\right\}=0
$$

Finally, Poisson bracket between matrix elements of $A$ is given by (60b) $\{A \otimes A\}=(A \otimes A) r_{a}+r_{a}(A \otimes A)+(\mathrm{Id} \otimes A) r_{21}(A \otimes \mathrm{Id})-(A \otimes \mathrm{Id}) r_{12}(\mathrm{Id} \otimes A)$ 


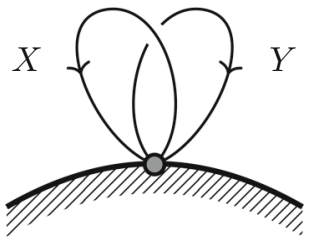

(A) Genus one pair

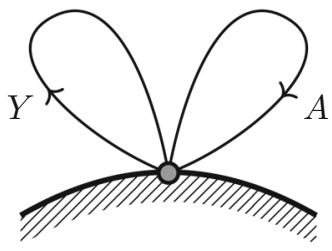

(в) Genus zero pair

Fig. 13. Relative order of half edges for pairs of closed arcs

By Leibnitz Identity we get for all $k \in \mathbb{N}$

$$
\begin{aligned}
\left\{A^{k} \otimes A\right\}= & \sum_{j=0}^{k-1}\left(A^{j} \otimes \mathrm{Id}\right)\{A \otimes A\}\left(A^{k-j-1} \otimes \mathrm{Id}\right) \\
= & \sum_{j=0}^{k-1}\left(\left(A^{j+1} \otimes A\right) r_{a}\left(A^{k-j-1} \otimes \mathrm{Id}\right)+\left(A^{j} \otimes \mathrm{Id}\right) r_{a}\left(A^{k-j} \otimes A\right)\right. \\
& \left.+\left(A^{j} \otimes A\right) r_{21}\left(A^{k-j} \otimes \mathrm{Id}\right)-\left(A^{j+1} \otimes \mathrm{Id}\right) r_{12}\left(A^{k-j-1} \otimes A\right)\right)
\end{aligned}
$$

Taking the partial trace with respect to the first component we have

$$
\begin{aligned}
\left\{\operatorname{Tr} A^{k}, A\right\} & =k \operatorname{Tr}_{1}\left(\left(A^{k} \otimes A\right) r_{a}+r_{a}\left(A^{k} \otimes A\right)+\left(A^{k} \otimes A\right) r_{21}-r_{12}\left(A^{k} \otimes A\right)\right) \\
& =\frac{k}{2} \operatorname{Tr}_{1}\left[A^{k} \otimes A, I\right] .
\end{aligned}
$$

From the invariance of $I$ and from the cyclic invariance of the trace we get

$$
(\operatorname{Tr} \otimes \mathrm{Id})\left(\left(A^{k} \otimes A\right) I\right)=(\operatorname{Tr} \otimes \mathrm{Id})\left(\left(A^{k-1} I(A \otimes A)\right)=(\operatorname{Tr} \otimes \operatorname{Id})\left(I\left(A^{k} \otimes A\right)\right)\right.
$$

This implies that the bracket $\left\{\operatorname{Tr} A^{k}, A\right\}$ vanishes.

Corollary 33. Let $(y, x)$ and $(y, a)$ be pairs of generators with relative order of half edges as shown on Fig. 13 and $H$ and $\pi_{V}$ as in the previous proposition. Denote by $A, X, Y \in \operatorname{Hom}\left(\pi_{1}\left(\Sigma, p_{1}, \ldots, p_{m}\right), G\right)$ the holonomies along the corresponding closed oriented arcs. We have

$$
\begin{aligned}
& \left\{H(Y), \pi_{V}(X)\right\}=\sum_{J}\left(e_{J}, \nabla H(A)\right) \pi_{V}\left(X e_{J}\right), \\
& \left\{H(Y), \pi_{V}(A)\right\}=\left\{H(Y), \pi_{V}(Y)\right\}=0 .
\end{aligned}
$$

Proof. Indeed, the Poisson bracket between $\operatorname{Tr} Y^{k}$ and $X_{i j}$ gets two contributions of the form (61a) and (61b) which correspond to the outgoing and the ingoing half edges of $X$ respectively. As a result, we obtain (64a).

On the other hand, the Poisson bracket between $\operatorname{Tr} Y^{k}$ and $A_{i j}$ is trivial due to (61b) because none of the half edges of $A$ appear in between half edges of $Y$. Similar logic applies to the bracket between $\operatorname{Tr} A^{k}$ and $Y_{i j}$. 
Note that the Hamilton flow given by $H(Y)$ preserves matrix element functions of the group commutator $\pi_{V}\left(X Y X^{-1} Y^{-1}\right)$.

Theorem 34. Let $\Sigma$ be an oriented surface with $b>0$ boundary components and $C=C_{1} \sqcup \ldots \sqcup C_{k}$ be a disjoint union of pairwise nonintersecting simple closed curves in $\Sigma$ as in Theorem 17, $H: G \rightarrow \mathbb{C}$ be a central function and $\pi_{V}: G \rightarrow \operatorname{End}(V)$ be a finite dimensional representation. Fix a choice of marked points $p_{1}, \ldots, p_{m} \in \partial \Sigma$ and generators of $\pi_{1}\left(\Sigma, p_{1}, \ldots, p_{m}\right)$ as in Sect. 4.1 and denote by $A_{i}, E_{i}, X_{l}, Y_{l}, Z_{o} \in$ $\operatorname{Hom}\left(\pi_{1}\left(\Sigma, p_{1}, \ldots, p_{m}\right), G\right)$ the holonomies along arcs $a_{i}, e_{i}, x_{l}, y_{l}, z_{o}$, then

$$
\begin{aligned}
& \left\{H\left(A_{i}\right), \pi_{V}\left(E_{j}\right)\right\}= \begin{cases}\sum_{J}\left(e_{J}, \nabla H\left(A_{i}\right)\right) \pi_{V}\left(E_{j} e_{J}\right), & i=j, \\
0, & i \neq j,\end{cases} \\
& \left\{H\left(A_{i}\right), \pi_{V}\left(X_{l}\right)\right\}=\left\{H\left(A_{i}\right), \pi_{V}\left(Y_{l}\right)\right\}=\left\{H\left(A_{i}\right), \pi_{V}\left(Z_{o}\right)\right\}=0, \\
& \left\{H\left(Y_{l}\right), \pi_{V}\left(X_{s}\right)\right\}= \begin{cases}\sum_{J}\left(e_{J}, \nabla H\left(Y_{l}\right)\right) \pi_{V}\left(X_{s} e_{J}\right), & l=s, \\
0, & l \neq s,\end{cases} \\
& \left\{H\left(Y_{l}\right), \pi_{V}\left(A_{i}\right)\right\}=\left\{H\left(Y_{l}\right), \pi_{V}\left(E_{i}\right)\right\}=\left\{H\left(Y_{l}\right), \pi_{V}\left(Z_{o}\right)\right\}=0 .
\end{aligned}
$$

Particular cases of Theorem 34 already appeared in the literature. For example, these brackets were computed in [FR99] for $G=S L(N, \mathbb{C})$ and a torus with one boundary component.

The following Corollary generalizes classical result from $\S 4$ of [Gol86] to the case of surfaces with several marked points on different boundary components and arbitrary number of separating cycles:

Corollary 35. Fix $i, 1 \leqslant i \leqslant r$ and a central function $H: G \rightarrow \mathbb{C}$. Then the Hamiltonian flow generated by the function $H$ evaluated on a cycle $C_{i} \in \Sigma$ is given by the following expressions

- When $C_{i} \in \partial \Sigma_{a} \cap \partial \Sigma_{b}$ separates a pair of distinct connected components of $\Sigma \backslash C$ we have

$$
\begin{aligned}
& E_{j}(t)= \begin{cases}E_{j} \exp \left(t \nabla H\left(A_{i}\right)\right), & i=j, \\
E_{j}, & i \neq j,\end{cases} \\
& X_{l}(t)=X_{l}, \quad Y_{l}(t)=Y_{l}, \\
& A_{j}(t)=A_{j}, \quad Z_{o}(t)=Z_{o} .
\end{aligned}
$$

- Similarly, when $C_{l}$ is a nonseparating cycle which belongs to the boundary of a single connected component of $\Sigma \backslash C$ the Hamiltonian flow generated by $H$ evaluated at the holonomy along $C_{l}$ is given by

$$
\begin{aligned}
X_{s}(t) & = \begin{cases}X_{s} \exp \left(t \nabla H\left(Y_{l}\right)\right), & l=s, \\
X_{s}, & l \neq s,\end{cases} \\
A_{j}(t) & =A_{j}, \quad E_{j}(t)=E_{j} \\
Y_{l}(t) & =Y_{l}, \quad Z_{o}(t)=Z_{o} .
\end{aligned}
$$

Remark 36. Let $\psi_{t}$ is the evolution on the algebra of functions on $\operatorname{Hom}\left(\pi_{1}\left(\Sigma, p_{1}, \ldots\right.\right.$, $\left.\left.p_{m}\right), G\right)$ generated by $H$, i.e.

$$
\psi_{t}^{H}(F)=F+\sum_{n \geq 1} \frac{t^{n}}{n !}\{H,\{H, \ldots,\{H, F\} \ldots\}\} .
$$


If the functions $H_{1}$ and $H_{2}$ Poisson commute then $\psi_{t_{1}}^{H_{1}} \psi_{t_{2}}^{H_{2}}=\psi_{t_{2}}^{H_{2}} \psi_{t_{1}}^{H_{1}}$. If $H_{1}, \ldots, H_{k}$ is a complete set of independent functions on holonomies along $C$, their joint flow lines generate ange variables on their level surfaces.

Remark 37. It is worth noting that Theorem 34 along with Corollary 35 can be used for computations of Hamilton flows on decorated moduli spaces, namely on the quotient of $\operatorname{Hom}\left(\pi_{1}\left(\Sigma, p_{1}, \ldots, p_{m}\right), G\right)$ by a proper subgroup of $G^{\times m}$. Examples of decorated character varieties include monodromy data of Painleve equations [CMR17,Boa18].

\section{Particular Cases}

Here we will describe Poisson algebras $\mathcal{O}\left[\mathcal{M}_{\Sigma}^{G}\right]$ and corresponding superintegrable systems explicitly in few low dimensional examples when $\Sigma$ is a torus with one or two boundary components. Writing out examples inevitably brings us to the problem of an explicit description of the invariant ring $\mathcal{O}\left[\mathcal{M}_{\Sigma}^{G}\right]$. For a simple linear algebraic group $G$ such invariant rings are always finitely generated, yet the number of generators grows much faster than the dimension of the corresponding moduli space. Here we describe subalgebras $Z_{\partial \Sigma} \subset B_{C} \subset J_{\Sigma \backslash C} \subset \mathcal{O}\left[\mathcal{M}_{\Sigma}^{G}\right]$ and their Poisson brackets internally, in terms of generators of the invariant ring $\mathcal{O}\left[\mathcal{M}_{\Sigma}^{G}\right]$ for some simple examples.

5.1. Torus with one boundary component, $G=S L(2, \mathbb{C})$. Let $\Sigma_{1,1}$ denote the torus with one boundary component. Choose a marked point $p_{1} \in \partial \Sigma_{1,1}$. The fundamental group $\pi_{1}\left(\Sigma_{1,1}, p_{1}\right)$ is freely generated by a pair of arcs $X, Y$ along the equator and meridian of the torus. Let $\Gamma$ be the ribbon graph associated to such choice of generators. The data of $\Gamma$ is encoded by an ordered set

$$
S_{1}=\left\{X^{-1}, Y, X, Y^{-1}\right\}
$$

of half-edges adjacent to $p_{1}$ as shown on Fig. 13a. In (65) we have labelled half-edges by generators $X, Y$ of $\pi_{1}\left(\Sigma_{1,1}, p_{1}\right)$ and their inverses $X^{-1}, Y^{-1}$ according to the convention introduced in the last paragraph of Sect. 4.1. Namely, we label an outgoing half-edge by the first power of the corresponding generator, while we label an ingoing edge by the inverse of the corresponding generator.

Moduli space The moduli space of flat $S L(2, \mathbb{C})$-connections on a once punctured torus has dimension $n=3$. We will use notation

$$
\tau_{A}=\operatorname{Tr}(A)
$$

for the trace of a matrix $A$.

The coordinate ring of the moduli space in this example is a free commutative algebra with three generators

$$
\mathcal{O}\left[\mathcal{M}_{\Sigma_{1,1}}^{S L(2, \mathbb{C})}\right]=\mathbb{C}\left[\tau_{X}, \tau_{Y}, \tau_{X Y}\right]
$$


where $X, Y \in S L(2, \mathbb{C})$ are elements representing standard $a$ and $b$ cycles. The Poisson bracket between these coordinate functions are:

$$
\begin{aligned}
\left\{\tau_{X}, \tau_{Y}\right\} & =-\tau_{X Y}+\frac{1}{2} \tau_{X} \tau_{Y}, \\
\left\{\tau_{X}, \tau_{X Y}\right\} & =\tau_{Y}-\frac{1}{2} \tau_{X} \tau_{X Y}, \\
\left\{\tau_{Y}, \tau_{X Y}\right\} & =-\tau_{X}+\frac{1}{2} \tau_{Y} \tau_{X Y} .
\end{aligned}
$$

This is a rank 2 Poisson structure. The center of $\mathcal{O}\left[\mathcal{M}_{\Sigma_{1,1}}^{S L(2, \mathbb{C})}\right]$ is generated by a single Casimir function

$$
Z=\mathbb{C}[z], \quad z=\tau_{X}^{2}+\tau_{Y}^{2}+\tau_{X Y}^{2}-\tau_{X} \tau_{Y} \tau_{X Y}=\operatorname{Tr}\left(X Y X^{-1} Y^{-1}\right)
$$

Superintegrable system Choose cycle $C$ defining the system to be $Y$ and the Hamiltonian $H=\tau_{Y}$. From (29) we obtain a chain of subgroups

$$
\pi_{1}(C)=\left\langle X Y X^{-1} Y^{-1}\right\rangle \quad \subset \pi_{1}\left(\Sigma_{1,1} \backslash C, p\right)=\left\langle Y, X Y X^{-1}\right\rangle \quad \subset \quad \pi_{1}\left(\Sigma_{1,1}, p\right)=\langle X, Y\rangle .
$$

Here and below we write $\left\langle a_{1}, \ldots, a_{n}\right\rangle$ for a free group generated by $a_{1}, \ldots, a_{n}$.

The algebra of first integrals is then generated by three elements, two of which coincide

$$
J=\mathcal{O}\left[\operatorname{Hom}\left(\pi_{1}\left(\Sigma_{1,1} \backslash C, p\right)\right)\right]=\mathbb{C}\left[\tau_{X Y X^{-1}}, \tau_{Y^{-1}}, \tau_{X Y X^{-1} Y^{-1}}\right]=\mathbb{C}[z, H] .
$$

As a result, we obtain the following chain of subalgebras of the coordinate ring

$$
Z \quad \subset \quad B=\mathbb{C}[z, H] \quad \subset \quad J=\mathbb{C}[z, H] \quad \subset \quad \mathcal{O}\left[\mathcal{M}_{\Sigma_{1,1}}^{S L(2, \mathbb{C})}\right] .
$$

In this case the algebra of Hamiltonians coincide with teh algebra of first integrals and therefore the system is Liouville integrable.

Mapping class group action Consider the mapping class group $\operatorname{Mod}\left(\Sigma_{1,1,0}\right)$ (relative to the boundary) of the torus with one boundary component and no punctures. It contains two left Dehn twists along the $X$ and $Y$ cycles which satisfy the braid relation and are acting on our generators as follows:

$$
D_{X}:\left\{\begin{array}{l}
X \mapsto X, \\
Y \mapsto Y X^{-1},
\end{array} \quad D_{Y}:\left\{\begin{array}{l}
X \mapsto X Y, \\
Y \mapsto Y,
\end{array} \quad D_{X} D_{Y} D_{X}=D_{Y} D_{X} D_{Y}=\left\{\begin{array}{l}
X \mapsto X Y X^{-1}, \\
Y \mapsto X^{-1}
\end{array}\right.\right.\right.
$$

This defines a pair of Poisson automorphisms $\mathcal{D}_{X}, \mathcal{D}_{Y}$ of the coordinate $\operatorname{ring} \mathcal{O}\left[\mathcal{M}_{\Sigma_{1,1}}^{S L(2, \mathbb{C})}\right]$

$$
\mathcal{D}_{X}=\left\{\begin{array}{l}
\tau_{X} \mapsto \tau_{X}, \\
\tau_{Y} \mapsto-\tau_{X Y}+\tau_{X} \tau_{Y}, \\
\tau_{X Y} \mapsto \tau_{Y},
\end{array} \quad \mathcal{D}_{Y}=\left\{\begin{array}{l}
\tau_{X} \mapsto \tau_{X Y}, \\
\tau_{Y} \mapsto \tau_{Y}, \\
\tau_{X Y} \mapsto-\tau_{X}+\tau_{Y} \tau_{X Y},
\end{array}\right.\right.
$$

Note that action of $\operatorname{Mod}\left(\Sigma_{1,1,0}\right)$ on the character variety factors through the action of the Mapping Class Group of a torus with one puncture $\operatorname{Mod}\left(\Sigma_{1,0,1}\right) \simeq S L(2, \mathbb{Z})$.

Poisson automorphisms (68) define a family of isomorphic integrable systems associated to nonseparating cycles on $\Sigma_{1,1}$. 
Table 1. Brackets between generators of $\mathcal{O}\left[\mathcal{M}_{\Sigma_{1,2}}^{S L(2)}\right]$

\begin{tabular}{llll}
\hline & $\tau_{X}$ & $\tau_{Y}$ & $\tau_{X Y}$ \\
\hline$\tau_{X}$ & 0 & $*$ & $*$ \\
$\tau_{Y}$ & $\frac{\tau_{X} \tau_{Y}}{2}-\tau_{X Y}$ & 0 & $*$ \\
$\tau_{X Y}$ & $\tau_{Y}-\frac{1}{2} \tau_{X} \tau_{X Y}$ & $\frac{1}{2} \tau_{Y} \tau_{X Y}-\tau_{X}$ & 0 \\
$\tau_{X Z}$ & 0 & $\tau_{X Y Z}-\frac{1}{2} \tau_{Y} \tau_{X Z}-\tau_{Y} \tau_{Z}+\frac{1}{2} \tau_{X Y} \tau_{X Z}+\tau_{Y Z}$ \\
$\tau_{Y Z}$ & $\tau_{X} \tau_{Y} \tau_{Z}-\tau_{X Y} \tau_{Z}-\tau_{Y} \tau_{X Z}-\frac{1}{2} \tau_{X} \tau_{Y Z}+\tau_{X Y Z} Z_{X}$ & $\tau_{X} \tau_{Z}-\tau_{X Z}-\frac{1}{2} \tau_{X Y} \tau_{Y Z}$ \\
$\tau_{X Y Z} \tau_{Y} \tau_{Z}-\tau_{X Y} \tau_{X Z}-\tau_{Y Z}+\frac{1}{2} \tau_{X} \tau_{X Y Z}$ & $\frac{1}{2} \tau_{Y} \tau_{X Y Z}-\tau_{X Z} 0$ \\
\multicolumn{1}{l}{$\tau_{X Z}$} & $\tau_{Y Z}$ & 0 \\
$\tau_{Y Z}$ & $\tau_{X Y}+\frac{1}{2} \tau_{X Z} \tau_{Y Z}-\tau_{Z} \tau_{X Y Z}$ & 0 & $-\tau_{X}+\tau_{Y} \tau_{X Y}-\tau_{Y} \tau_{Z} \tau_{X Y Z}+\frac{1}{2} \tau_{Y Z} \tau_{X Y Z}+\tau_{Z} \tau_{X Z}$ \\
$\tau_{X Y Z} \tau_{Y}-\frac{1}{2} \tau_{X Z} \tau_{X Y Z}$ & &
\end{tabular}

5.2. Torus with two boundary components, $G=S L(2, \mathbb{C})$. The coordinate ring of the moduli space has dimension $n=6$ and the coordinate ring is generated by 7 polynomials subject to the single relation

$$
\mathcal{O}\left[\mathcal{M}_{\Sigma_{1,2}}^{S L(2, \mathbb{C})}\right]=\frac{\mathbb{C}\left[\tau_{X}, \tau_{Y}, \tau_{Z}, \tau_{X Y}, \tau_{X Z}, \tau_{Y Z}, \tau_{X Y Z}\right]}{\left(\tau_{X Y Z}^{2}+\mu_{1} \tau_{X Y Z}+\mu_{0}\right)} .
$$

Here $\tau_{A}$ stands for $\operatorname{Tr}(A)$ and

$$
\begin{aligned}
\mu_{1}= & \tau_{Z} \tau_{X Y}+\tau_{Y} \tau_{X Z}+\tau_{X} \tau_{Y Z}-\tau_{X} \tau_{Y} \tau_{Z}, \\
\mu_{0}= & \tau_{X} \tau_{Y} \tau_{X Y}+\tau_{Y} \tau_{Z} \tau_{Y Z}+\tau_{X} \tau_{Z} \tau_{X Z}-\tau_{X Y} \tau_{X Z} \tau_{Y Z} \\
& -\tau_{X Y}^{2}-\tau_{X Z}^{2}-\tau_{Y Z}^{2}-\tau_{X}^{2}-\tau_{Y}^{2}-\tau_{Z}^{2}+4
\end{aligned}
$$

Poisson brackets between generators are summarized in Table 1, where we have omitted generator $\tau_{Z}$ which belongs to the Poisson center of $\mathcal{O}\left[\mathcal{M}_{\Sigma_{1,2}}^{S L(2, \mathbb{C})}\right]$.

The Poisson center $Z$ of the coordinate ring has Krull dimension $\operatorname{dim} Z=2$ and has two algebraically independent Casimir elements corresponding to traces of monodromies around each of the boundary components

$$
z_{1}=\tau_{Z}, \quad z_{2}=\operatorname{Tr}\left(X Y X^{-1} Y^{-1} Z\right)=-\tau_{Y} \tau_{X Y} \tau_{X Z}+\tau_{X Y} \tau_{X Y Z}+\tau_{X} \tau_{X Z}-\tau_{Y} \tau_{Y Z}+\tau_{Y}^{2} \tau_{Z}-\tau_{Z}
$$

5.2.1. A superintegrable system associated to nonseparating cycle Consider a superintegrable system given by a nonseparating cycle homotopic to $Y$, from (29) we get a chain of subgroups

$$
\pi_{1}(C)=\langle Y\rangle \quad \subset \quad \pi_{1}\left(\Sigma_{1,2}, p \mid C\right)=\left\langle Y, X Y X^{-1}, Z\right\rangle \quad \subset \quad \pi_{1}\left(\Sigma_{1,2}, p\right)=\langle X, Y, Z\rangle
$$

Coordinate ring of the character variety of the smallest subgroup is generated by a single hamiltonian $H=\tau_{Y}$. At the same time, the coordinate ring of the character variety of 
the middle subgroup in (69) has the following presentation ${ }^{17}$

$$
\begin{aligned}
J & =\mathcal{O}\left[\operatorname{Hom}\left(\pi_{1}\left(\Sigma_{1,1}, p \mid C\right), S L(2)\right)\right]^{S L(2)} \\
& =\frac{\mathbb{C}\left[\tau_{Y}, \tau_{Z}, \tau_{Y Z}, \tau_{X Y X^{-1} Z}, \tau_{Y X Y X^{-1}}, \tau_{X Y X^{-1} Y Z}\right]}{\left(\tau_{X Y X^{-1} Y Z}^{2}+\lambda_{1} \tau_{X Y X^{-1} Y Z}+\lambda_{0}\right)},
\end{aligned}
$$

where

$$
\begin{aligned}
\lambda_{1}= & \tau_{Y} \tau_{X Y X^{-1} Z}+\tau_{Z} \tau_{Y X Y X^{-1}}+\tau_{Y} \tau_{Y Z}-\tau_{Y}^{2} \tau_{Z}, \\
\lambda_{0}= & \tau_{Y}^{2} \tau_{Y X Y X^{-1}}+\tau_{Y} \tau_{Z} \tau_{X Y X^{-1} Z}-\tau_{Y Z} \tau_{Y X Y X^{-1}} \tau_{X Y X^{-1} Z}+\tau_{Y} \tau_{Z} \tau_{Y Z} \\
& -\tau_{Y X Y X^{-1}}^{2}-\tau_{X Y X^{-1} Z}^{2}-\tau_{Y Z}^{2}-2 \tau_{Y}^{2}-\tau_{Z}^{2} .
\end{aligned}
$$

Subalgebra $J \subset \mathcal{O}\left[\mathcal{M}_{\Sigma_{1,2}}^{S L(2)}\right]$ is closed under the Poisson bracket. Explicitly, all nonzero brackets between generators are given by

$$
\begin{aligned}
& \left\{\tau_{X Y X^{-1} Z}, \tau_{Y X Y X^{-1}}\right\}=\tau_{Y} \tau_{Z}-2 \tau_{Y Z}-\tau_{X Y X^{-1} Z} \tau_{Y X Y X^{-1}}+\tau_{Y} \tau_{X Y X^{-1} Y Z} \\
& \left\{\tau_{Y Z}, \tau_{Y X Y X^{-1}}\right\}=\tau_{Z} \tau_{Y}^{3}-\tau_{Y Z} \tau_{Y}^{2}-\tau_{X Y X^{-1} Z^{-}} \tau_{Y}^{2}-\tau_{Z} \tau_{Y}-\tau_{Z} \tau_{Y X Y X^{-1}} \tau_{Y} \\
& +\tau_{X Y X^{-1} Y Z} \tau_{Y}+2 \tau_{X Y X^{-1} Z}+\tau_{Y Z} \tau_{Y X Y X^{-1}} \\
& \left\{\tau_{Y Z}, \tau_{X Y X^{-1} Z}\right\}=\tau_{Y}^{2}-\tau_{Y Z} \tau_{X Y X^{-1} Z}-2 \tau_{Y X Y X^{-1}}+\tau_{Z} \tau_{X Y X^{-1} Y Z} \\
& \left\{\tau_{X Y X^{-1} Y Z}, \tau_{Y X Y X^{-1}}\right\}=\tau_{Z} \tau_{Y}^{2}+\tau_{X Y X^{-1} Y Z} \tau_{Y}^{2}-2 \tau_{Y Z} \tau_{Y}-\tau_{X Y X^{-1} Z \tau_{Y X X^{-1}}} \tau_{Y} \\
& \left\{\tau_{X Y X^{-1} Y Z}, \tau_{Y Z}\right\}=-\tau_{Y}^{3}+\tau_{Y Z} \tau_{X Y X^{-1} Z} \tau_{Y}+2 \tau_{Y X Y X^{-1}} \tau_{Y}-\tau_{Z} \tau_{X Y X^{-1} Y Z} \tau_{Y}
\end{aligned}
$$

The algebra of first integrals (70) has Krull dimension 5. One can choose its maximal algebraically independent subset to contain two Casimir functions

$$
z_{1}=\tau_{Z}, \quad z_{2}=\tau_{Y} \tau_{X Y X^{-1} Z}-\tau_{X Y X^{-1} Y Z}
$$

one Hamiltonian

$$
H=\tau_{Y},
$$

and two more first integrals

$$
g_{1}=\tau_{Y Z}=\tau_{Y Z}, \quad g_{2}=\tau_{X Y X^{-1} Y}=\tau_{X Y}^{2}+\tau_{X} \tau_{Y} \tau_{X Y}-\tau_{X}^{2}+2
$$

17 Finding a generating set together with transcendence basis for the corresponding field of fractions is always straightforward in the case of all rings involved in our paper. However, it is much more complicated to find a complete set of relations outside of the most elementary examples.

In this particular case, one can recall that $J$ is isomorphic to a quotient ring of $\mathcal{O}\left[M_{\Sigma_{0,4}}^{S L(2)}\right]$ modulo the relation that traces of monodromies around the fixed pair of boundary components are equal to each other. This allows one to resolve one of the generators in $\mathcal{O}\left[M_{\Sigma_{0,4}}^{S L(2)}\right]$ and obtain presentation (70). 
5.2.2. Separating cycle Now consider a superintegrable system given by a separating cycle homotopic to $X Y X^{-1} Y^{-1}$. As in the previous case we have two Casimir elements $z_{1}$ and $z_{2}$. Their generic level sets form a 2-parametric family of 4-dimensional symplectic manifolds. Choose the Hamiltonian on these phase spaces as

$$
H=\operatorname{Tr}\left(X Y X^{-1} Y^{-1}\right)=\tau_{X}^{2}+\tau_{Y}^{2}+\tau_{X Y}^{2}-\tau_{X} \tau_{Y} \tau_{X Y}-2 .
$$

From (51) we have the following algebra of first integrals

$$
\begin{aligned}
J & =J_{L} \otimes_{B} J_{R}, & B & =\mathbb{C}[H] \simeq \mathcal{O}[S L(2)]^{S L(2)}, \\
J_{L} & =\mathbb{C}\left[\tau_{X}, \tau_{Y}, \tau_{X Y}\right] \simeq \mathcal{O}\left[\mathcal{M}_{\Sigma_{1,1}}^{S L(2)}\right], & J_{R} & =\mathbb{C}\left[z_{1}, z_{2}, H\right] \simeq \mathcal{O}\left[\mathcal{M}_{\Sigma_{0,3}}^{S L(2)}\right] .
\end{aligned}
$$

Here $J_{R}$ is Poisson commutative, while $J_{L}$ is equipped with a Poisson bracket of generic rank 2 (see first three rows of Table 1). Algebra $J$ has Krull dimension 5. An example of maximal algebraically independent subset is $z_{1}, z_{2}, H, g_{1}=\tau_{X}, g_{2}=\tau_{Y}$, i.e. generators of the Poisson center, the Hamiltonian and two additional first integrals.

5.3. Torus with one boundary component, $G=S L(3, \mathbb{C})$. The moduli space of flat $S L(3, \mathbb{C})$-connections on a once punctured torus has dimension $n=8$, the coordinate ring is generated by 9 polynomials subject to a single relation

$$
\mathcal{O}\left[\mathcal{M}_{\Sigma_{1,1}}^{S L(3)}\right]=\frac{\mathbb{C}\left[\tau_{X}, \tau_{Y}, \tau_{X^{-1}}, \tau_{Y^{-1}}, \tau_{Y X}, \tau_{Y X^{-1}}, \tau_{Y^{-1} X}, \tau_{Y^{-1} X^{-1}}, \tau_{X Y X^{-1} Y^{-1}}\right]}{\left(\tau_{X Y X^{-1} Y^{-1}}^{2}+\zeta_{1} \tau_{X Y X^{-1} Y^{-1}}+\zeta_{0}\right)},
$$

where $\zeta_{1}$ and $\zeta_{0}$ are polynomials in $\tau_{X}, \ldots \tau_{X^{-1} Y^{-1}}$ :

$$
\begin{aligned}
\zeta_{1}= & \operatorname{Tr}\left(Y X Y^{-1} X^{-1}\right) \\
= & \tau_{X} \tau_{X^{-1}}+\tau_{X} \tau_{Y^{-1}} \tau_{X^{-1}}-\tau_{X^{-1}} \tau_{Y^{-1}} \tau_{Y X}-\tau_{Y} \tau_{X^{-1}} \tau_{Y^{-1} X}-\tau_{X} \tau_{Y^{-1}} \tau_{Y X^{-1}} \\
& +\tau_{Y X^{-1}} \tau_{Y^{-1} X}-\tau_{X} \tau_{Y} \tau_{Y^{-1} X^{-1}}+\tau_{Y X} \tau_{Y^{-1} X^{-1}}-\tau_{X Y X^{-1} Y^{-1}}+\tau_{Y} \tau_{Y^{-1}}-3
\end{aligned}
$$

and

$$
\begin{aligned}
& \zeta_{0}=\tau_{Y} \tau_{Y^{-1}} \tau_{X}^{3}-\tau_{X}^{3}-\tau_{Y} \tau_{X^{-1}}^{2} \tau_{Y^{-1}} \tau_{X}^{2}-\tau_{Y}^{2} \tau_{Y X} \tau_{X}^{2}-\tau_{Y^{-1}} \tau_{Y X} \tau_{X}^{2}+\tau_{X^{-1}} \tau_{Y^{-1}} \tau_{Y^{-1}} \tau_{X}^{2} \\
& -\tau_{Y^{-1}}^{2} \tau_{Y^{-1}} \tau_{X}^{2}-\tau_{Y^{\prime}} \tau_{Y^{-1} X} \tau_{X}^{2}+\tau_{Y^{\prime}} \tau_{X^{-1}} \tau_{Y^{-1} X^{-1}} \tau_{X}^{2}-\tau_{Y^{-1}} \tau_{Y^{-1} X^{-1}} \tau_{X}^{2}+\tau_{X^{-1}} \tau_{Y^{-1}}^{3} \tau_{X} \\
& -\tau_{Y}^{2} \tau_{X^{-1}} \tau_{Y^{-1}}^{2} \tau_{X}+2 \tau_{Y} \tau_{Y X}^{2} \tau_{X}-\tau_{Y X} \tau_{Y X^{-1}}^{2} \tau_{X}+2 \tau_{Y^{-1}} \tau_{Y^{-1} X^{2}}^{2} \tau_{X}-\tau_{Y^{-1}} \tau_{Y^{-1} X^{-1}}^{2} \tau_{X} \\
& +\tau_{Y}^{3} \tau_{X^{-1}} \tau_{X}+6 \tau_{X^{-1}} \tau_{X}-\tau_{Y} \tau_{X^{-1}} \tau_{Y^{-1}} \tau_{X}+\tau_{X^{-1}}^{2} \tau_{Y^{-1}} \tau_{Y X} \tau_{X}-\tau_{Y}^{2} \tau_{Y^{-1}} \tau_{X} \\
& +\tau_{Y} \tau_{Y^{-1}}^{2} \tau_{Y^{-1}} \tau_{X}-3 \tau_{Y^{-1}} \tau_{Y^{-1}} \tau_{X}+\tau_{Y} \tau_{X^{-1}} \tau_{Y X} \tau_{Y^{-1}} \tau_{X}+\tau_{Y} \tau_{X^{-1}}^{2} \tau_{Y^{-1}} \tau_{X} \\
& +\tau_{Y} \tau_{Y^{-1}} \tau_{Y X} \tau_{Y^{-1}} \tau_{X}+3 \tau_{Y X} \tau_{Y^{-1} X} \tau_{X}-\tau_{X^{-1}} \tau_{Y^{-1}} \tau_{Y^{-1}} \tau_{X}-\tau_{Y^{-1}}^{2} \tau_{Y^{-1} X^{-1}} \tau_{X} \\
& -3 \tau_{Y} \tau_{Y^{-1} X^{-1}} \tau_{X}+\tau_{Y}^{2} \tau_{Y^{-1}} \tau_{Y^{-1} X^{-1}} \tau_{X}-\tau_{X^{-1}} \tau_{Y X} \tau_{Y^{-1} X^{-1}} \tau_{X}+\tau_{X^{-1}} \tau_{Y^{-1}} \tau_{Y^{-1} X^{\prime}} \tau_{Y^{-1} X^{-1}} \tau_{X} \\
& -\tau_{Y}^{3}-\tau_{X^{-1}}^{3}-\tau_{Y^{-1}}^{3}-\tau_{Y X}^{3}-\tau_{Y^{-1}}^{3}-\tau_{Y^{-1} X}^{3}-\tau_{Y^{-1} X^{-1}}^{3}+2 \tau_{Y} \tau_{X^{-1}} \tau_{Y X^{-1}}^{2}-\tau_{Y} \tau_{Y X} \tau_{Y^{-1} X}^{2} \\
& +2 \tau_{X^{-1}} \tau_{Y^{-1}} \tau_{Y^{-1} X^{-1}}^{2}-\tau_{Y} \tau_{Y^{-1}} \tau_{Y^{-1} X^{-1}}^{2}+\tau_{Y} \tau_{X^{-1}}^{3} \tau_{Y^{-1}}+6 \tau_{Y} \tau_{Y^{-1}}+\tau_{Y^{-1}} \tau_{X^{-1}} \tau_{Y^{-1}}^{2} \tau_{Y X} \\
& -\tau_{Y}^{2} \tau_{X^{-1}} \tau_{Y X}-3 \tau_{X^{-1}} \tau_{Y^{-1}} \tau_{Y X}-\tau_{Y^{\prime}}^{2} \tau_{X^{-1}}^{2} \tau_{Y_{X^{-1}}}-\tau_{X^{-1}} \tau_{Y X^{2}}^{2} \tau_{Y^{-1}}-\tau_{X^{-1}}^{2} \tau_{Y^{-1}} \tau_{Y^{-1}} \\
& -\tau_{Y^{-1}}^{2} \tau_{Y X} \tau_{Y^{-1}}+3 \tau_{Y} \tau_{Y X} \tau_{Y^{-1}}-\tau_{X^{-1}} \tau_{Y^{-1}}^{2} \tau_{Y^{-1} X}-\tau_{Y^{-1}} \tau_{Y X^{\prime}}^{2} \tau_{Y^{-1} X}-3 \tau_{Y} \tau_{X^{-1}} \tau_{Y^{-1} X} \\
& +\tau_{Y^{-1}}^{2} \tau_{X^{-1}} \tau_{Y^{-1}} \tau_{Y^{-1} X}-\tau_{X^{-1}}^{2} \tau_{Y X} \tau_{Y^{-1} X}-\tau_{Y} \tau_{Y^{-1}} \tau_{Y^{-1}} \tau_{Y^{-1} X}+6 \tau_{Y^{-1}} \tau_{Y^{-1} X} \\
& -\tau_{Y} \tau_{X^{-1}}^{2} \tau_{Y^{-1} X^{-1}}-\tau_{X^{-1}}^{2} \tau_{Y^{-1}}^{2} \tau_{Y^{-1} X^{-1}}-\tau_{Y^{-1}} \tau_{Y^{-1}}^{2} \tau_{Y^{-1} X^{-1}}-\tau_{X^{-1}} \tau_{Y^{-1} X^{-1}}^{2} \tau_{Y^{-1} X^{-1}}
\end{aligned}
$$




$$
\begin{aligned}
& -\tau_{Y} \tau_{Y^{-1}} \tau_{Y X} \tau_{Y^{-1} X^{-1}}+6 \tau_{Y X} \tau_{Y^{-1} X^{-1}}+3 \tau_{X^{-1}} \tau_{Y X^{-1}} \tau_{Y^{-1} X^{-1}}+\tau_{Y} \tau_{X^{-1}} \tau_{Y^{-1}} \tau_{Y^{-1}} \tau_{Y^{-1} X^{-1}} \\
& -\tau_{Y}^{2} \tau_{Y^{-1} X} \tau_{Y^{-1} X^{-1}}+3 \tau_{Y^{-1}} \tau_{Y^{-1}} \tau_{Y^{-1} X^{-1}}-\tau_{Y X} \tau_{Y^{-1}} \tau_{Y^{-1}} \tau_{Y^{-1} X^{-1}}-9
\end{aligned}
$$

For a proof, see for example Lemma 5 in [Law07].

Poisson brackets between generators are summarized in Table 2, where we have omitted generator $\tau_{X Y X^{-1} Y^{-1}}$ because it belongs to the Poisson center of $\mathcal{O}\left[\mathcal{M}_{\Sigma_{1,1}}^{S L(3, \mathbb{C})}\right]$, see [Law09].

Coordinate ring $\mathcal{O}\left[\mathcal{M}_{\Sigma_{1,1}}^{S L(3)}\right]$ has a Poisson center $Z$ of Krull dimension $\operatorname{dim} Z=2$. Two algebraically idependent Casimir elements: $z_{1}=\tau_{Y X Y^{-1} X^{-1}}$ and $z_{2}=\tau_{X Y X^{-1} Y^{-1}}$. The formula expressing $z_{1}$ in terms of generators is given in (73)

5.3.1. A superintegrable system Here we consider a superintegrable system defined by a single cycle homotopic to $X$. By (29) we have a chain of inclusions of subgroups of the fundamental group

$$
\pi_{1}(C)=\langle X\rangle \quad \subset \quad \pi_{1}\left(\Sigma_{1,1}, p \mid C\right)=\left\langle X, Y X Y^{-1}\right\rangle \quad \subset \quad \pi_{1}\left(\Sigma_{1,1}, p\right)=\langle X, Y\rangle .
$$

Choose two independent Hamiltonians in $\mathcal{O}\left[\operatorname{Hom}\left(\pi_{1}(C), S L(3)\right]^{S L(3)}\right.$ as

$$
H_{1}=\tau_{X}, \quad H_{2}=\tau_{X^{-1}}
$$

These two Poisson commuting Hamiltonians define a superintegrable system with the Poisson algebra of first integrals $J$ defined above. We can choose a maximal algebraically idependent subset in $J$ to be $z_{1}, z_{2}, H_{1}, H_{2}$ with two more integrals

$$
\begin{aligned}
& g_{1}=\operatorname{Tr}\left(Y X Y^{-1} X\right)=\tau_{X^{-1}}-\tau_{Y} \tau_{X^{-1}} \tau_{Y^{-1}}+\tau_{Y^{-1}} \tau_{Y X^{-1}}+\tau_{Y X} \tau_{Y^{-1} X}+\tau_{Y} \tau_{Y^{-1} X^{-1}} \\
& g_{2}=\operatorname{Tr}\left(Y X^{-1} Y^{-1} X^{-1}\right)=\tau_{X}-\tau_{X} \tau_{Y} \tau_{Y^{-1}}+\tau_{Y^{-1}} \tau_{Y X}+\tau_{Y} \tau_{Y^{-1} X}+\tau_{Y^{-1}} \tau_{Y^{-1} X^{-1}}
\end{aligned}
$$

\section{Conclusion}

6.1. The algebra of chord diagrams on a surface and "universal superintegrable systems". The algebra of chord diagrams is a universal model for the Poisson algebra of functions on moduli spaces of flat connections [AMR96], see also [T91] for the case of loop algebras on a surface, i.e. skein modules. Chord diagrams first appeared in the setting of finite type invariants of knots [V90] and in perturbative Chern-Simons invariants [BN91, K93]. They became an important tool in the related theory of Vassiliev invariants.

Let us recall some basic definitions. A chord diagram is a homotopy class of a graph on a surface which consists of solid lines and chords. Solid lines are oriented, cords are not oriented. They satisfy the analogue of Reidemeister relations (see [AMR96] for details) together with the so-called 4T-relation shown on Fig. 14.

The algebra of chord diagrams $C h(\Sigma)$ on a surface $\Sigma$ is a vector space spanned by $\mathbb{Z}$-linear combinations of chord diagrams with

- the commutative multiplication of $\left[D_{1}\right]$ and $\left[D_{2}\right]$ is defined as $\left[D_{1}\right]\left[D_{2}\right]=\left[D_{1} \sqcup\right.$ $\left.D_{2}\right]$, i.e. as the homotopy class of their disjoint union. T 


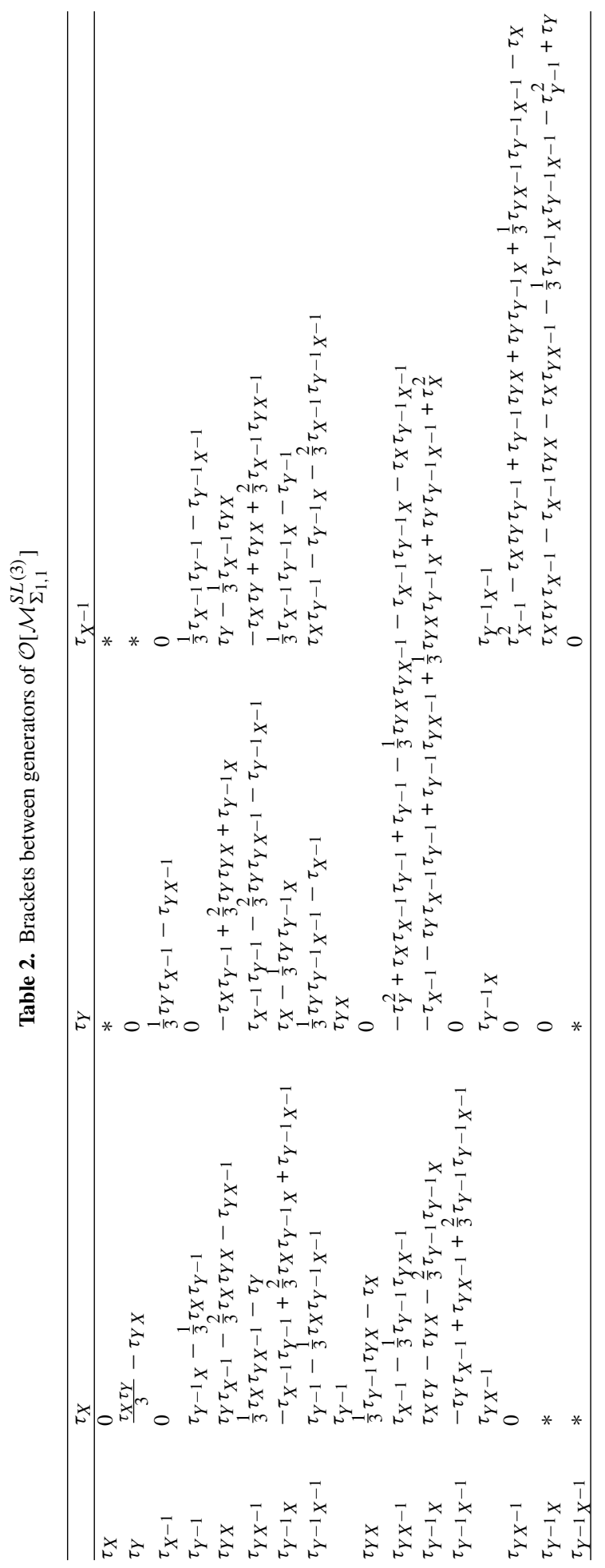



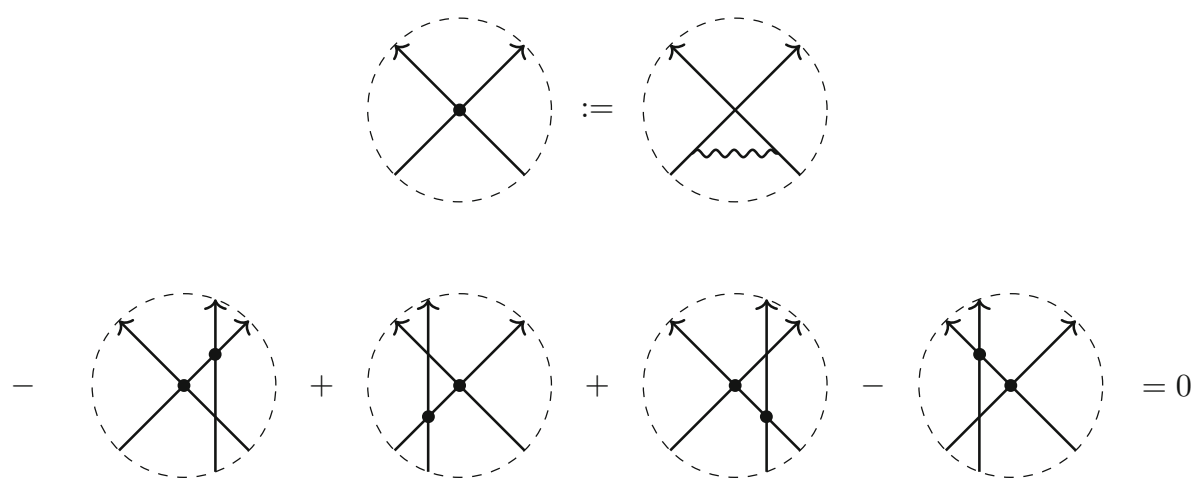

Fig. 14. $4 T$ relation in chord diagrams

- The Poisson bracket is a "universal" version of the Atiyah and Bott Poisson bracket. For two chord diagrams $D_{1}$ and $D_{2}$ their Poisson bracket is

$$
\left\{\left[D_{1}\right],\left[D_{2}\right]\right\}=\sum_{p \in D_{1} \cap D_{2}} \sum_{p \in D_{1} \cap D_{2}} \epsilon(p)\left[D_{1} \#_{p} D_{2}\right]
$$

where the sum is taken over the intersection points of representatives $D_{1}$ and $D_{2}$ and $D_{1} \#_{p} D_{2}$ is the result of the insertion of a chord in a small vicinity of point $p$. It is clear that the right side does not depend on the choice of representatives and on where the extra cord in inserted.

The Poisson center of $Z(\Sigma) \subset C h(\Sigma)$ is, conjecturally, generated by chord diagrams which are contractible to the boundary of $\Sigma$.

To define a conjecturally superintegrable system on $C h(\Sigma)$ choose a system of simple curves on the surface. Define the subalgebra of Hamiltonians $B(C)$ and the subalgebra of chord diagrams which are contractible to $C$ and the subalgebra of first integrals $J(C)$ and the subalgebra of chord diagrams which can be separated from $C$. We have a natural inclusion of Poisson subalgebras:

$$
Z(\Sigma) \subset B(C) \otimes Z(\Sigma) \subset J(C) \subset C h(\Sigma)
$$

We conjecture that this chain of inclusions define a superintegrable systems. In this infinite dimensional setting the superintegrability means that the space of all nontrivial Poisson derivations $\operatorname{Der}_{J(C)}(C h(\Sigma))$ of $C h(\Sigma)$ which act trivially on $J(C)$ is generated as an $C h(\Sigma)$-module by Hamilton derivations corresponding to elements from $B(C)$.

6.2. Quantization and the algebra of links in a cylinder. The natural question about any integrable system is how to quantize it. In the case of superintegrable systems on chord diagrams there is a natural quantization.

There is a natural associative algebra that can be naturally associated to a surface. The his is the algebra of links, or linked graphs, in $\Sigma \times I$ where $I$ is an interval. This algebra, which we will denote $A(\Sigma)$ is the space of $\mathbb{Z}$-linear combinations of links in $\Sigma \times I$. The natural associative multiplication on this space is the "placing of one link on the top of the other":

$$
\left[L_{1}\right] *\left[L_{2}\right]=\left[L_{1} \sqcup L_{2}\right]
$$


where in the right side of this equation we assume that $L_{1} \subset \Sigma[1,1 / 2]$ and $L_{2} \subset$ $\Sigma \times[1 / 2,0]$. here $[L]$ is the topological link which the isotopy class of a geometrical link $L: S^{1 \times n} \rightarrow \Sigma \times I$.

The algebra $A(\Sigma)$ has a natural filtration with $C h(\Sigma)$ being isomorphic to its associated graded algebra. In this sense $A(\Sigma)$ is the quantization of $C h(\Sigma)$.

Finite dimensional representations of the algebra $A(\Sigma)$ can be easily constructed using [RT90]. Such representations use representation theory of $U_{q}(\mathfrak{g})$ at roots of unity. We will present details in a separate publication.

For generic $q$, the quantization can be done by quantizing Fock and Rosly brackets by $R$-matrices, see for example [AGS95,AS96]. It is worth noting that a closely related question for the extended moduli space with the choice of flags at marked points on the boundary can be studied by means of Cluster Algebras [FG06], see for example [SS17, GSV18, CMR17] and references therein.

Acknowledgement. N.R. is grateful to J. Stokman for numerous discussions. S.A. is grateful to V. Roubtsov and L. Feher for useful remarks. The work of N.R. was partially supported by Grants NSF DMS-1601947 and RSF-18-11-00-297. The work of S.A. was partially supported by RSF-18-01-00926 and 19-51-50008-YaF.

Open Access This article is licensed under a Creative Commons Attribution 4.0 International License, which permits use, sharing, adaptation, distribution and reproduction in any medium or format, as long as you give appropriate credit to the original author(s) and the source, provide a link to the Creative Commons licence, and indicate if changes were made. The images or other third party material in this article are included in the article's Creative Commons licence, unless indicated otherwise in a credit line to the material. If material is not included in the article's Creative Commons licence and your intended use is not permitted by statutory regulation or exceeds the permitted use, you will need to obtain permission directly from the copyright holder. To view a copy of this licence, visit http://creativecommons.org/licenses/by/4.0/.

Publisher's Note Springer Nature remains neutral with regard to jurisdictional claims in published maps and institutional affiliations.

\section{References}

[AGS95] Alekseev, A., Grosse, H., Schomerus, V.: Combinatorial quantization of the Hamiltonian ChernSimons theory I. Commun. Math. Phys. 172(2), 317-358 (1995)

[AK00] Alekseev, A., Kosmann-Schwarzbach, Y.: Manin pairs and moment maps. J. Differ. Geom. 56(1), 133-165 (2000)

[AKM02] Alekseev, A., Kosmann-Schwarzbach, Y., Meinrenken, E.: Quasi-Poisson manifolds. Can. J. Math. 54(1), 3-29 (2002)

[AMM98] Alekseev, A., Malkin, A., Meinrenken, E.: Lie group valued moment maps. J. Differ. Geom. 48(3), 445-495 (1998)

[AR] Arthamonov, S., Roubtsov, V.: To appear

[AS96] Alekseev, A., Schomerus, V.: Representation theory of Chern-Simons observables. Duke Math. J. 85(2), 447-510 (1996)

[AO19] Arutyunov, G., Olivucci, E.: Hyperbolic spin Ruijsenaars-Schneider model from Poisson reduction. arXiv: 1906.02619

[AB83] Atiyah, M., Bott, R.: The Yang-Mills equations over Riemann surfaces. Philos. Trans. R. Soc. Lond. A 308(1505), 523-615 (1983)

[AMR96] Andersen, J., Mattes, J., Reshetikhin, N.: The Poisson structure on the moduli space of flat connections and chord diagrams. Topology 35(4), 1069-1083 (1996)

[A97] Audin, M.: In Gauge Theory and Symplectic Geometry. Lectures on Gauge Theory and Integrable Systems, pp. 1-48. Springer, Dordrecht (1997)

[BN91] Bar-Natan, D.: Perurbative aspects of the Chern-Simons topological quantum field theory. PhD thesis, Princeton University, Princeton (1991)

[BD82] Belavin, A., Drinfeld, V.: Solutions of the classical Yang-Baxter equation for simple Lie algebras. Funct. Anal. Appl. 16(3), 159-180 (1982)

[BG93] Biswas, I., Guruprasad, K.: Principal bundles on open surfaces and invariant functions on lie groups. Int. J. Math. 4(04), 535-544 (1993) 
[Boa18] Boalch, Ph.: Wild character varieties, points on the Riemann sphere and Calabi's examples. Adv. Stud. Pure Math. 76, 67-94 (2018)

[CF18] Chalykh, O., Fairon, M.: On the Hamiltonian formulation of the trigonometric spin RuijsenaarsSchneider system. Lett. Math. Phys. 110(11), 2893-2940 (2020)

[CMR17] Chekhov, L., Mazzocco, M., Rubtsov, V.: Algebras of quantum monodromy data and decorated character varieties. Int. Math. Res. Not. 2017(24), 7639-7691 (2017)

[FK11] Fehér, L., Klimčík, C.: Self-duality of the compactified Ruijsenaars-Schneider system from quasiHamiltonian reduction. Nucl. Phys. B 860(3), 464-515 (2012)

[FK13] Fehér, L., Klimčík, C.: The Ruijsenaars self-duality map as a mapping class symplectomorphism, In: Lie Theory and Its Applications in Physics, IX International Workshop, pp. 423-437. Springer (2013). arXiv:1203.3300

[FG06] Fock, V., Goncharov, A.: Moduli spaces of local systems and higher Teichmüller theory. Publ. Math. de l'IHÉS 103, 1-211 (2006)

[FGNR00] Fock, V., Gorsky, A., Nekrasov, N., Rubtsov, V.: Duality in integrable systems and gauge theories. JHEP 07, 028 (2000)

[F35] Fock, V.: Zur theorie des wasserstoffatoms. Z. Phys. 98, 145 (1935)

[FR93] Fock, V., Rosly, A.: Flat connections and polyubles. Theor. Math. Phys. 95(2), 526-534 (1993)

[FR99] Fock, V., Rosly, A.: Poisson structure on moduli of flat connections on Riemann surfaces and $r$-matrix. In: Moscow Seminar in Mathematical Physics. American Mathematical Soc., pp. 67-86 (1999)

[FMSW65] Frish, J., Mandrosov, V., Smorodinsky, Y., Uhlir, M., Winternitz, P.: On higher symmetries in quantum mechanics. Phys. Lett. 16, 354-356 (1965)

[GSV18] Gekhtman, M., Shapiro, M., Vainshtein, A.: Drinfeld double of $G L_{n}$ and generalized cluster structures. Proc. Lond. Math. Soc. 116(3), 429-484 (2018)

[GN94] Gorsky, A., Nekrasov, N.: Hamiltonian systems of Calogero type and two dimensional Yang-Mills theory. Nucl. Phys. B 414, 213-238 (1994)

[GN95] Gorsky, A., Nekrasov, N.: Relativistic Calogero-Moser Model as a gauged WZW model. Nucl. Phys. B 436, 582 (1995)

[GHJW97] Guruprasad, K., Huebschmann, J., Jeffrey, L., Weinstein, A.: Group systems, groupoids, and moduli spaces of parabolic bundles. Duke Math. J. 89(2), 377-412 (1997)

[Gol86] Goldman, W.: Invariant functions on Lie groups and Hamiltonian flows of surface group representations. Invent. Math. 85(2), 263-302 (1986)

[H87] Hitchin, N.: Stable bundles and integrable systems. Duke Math. J. 54(1), 91-114 (1987)

[JW92] Jeffrey, L., Weitsman, J.: Bohr-Sommerfeld orbits in the moduli space of flat connections and the Verlinde dimension formula. Commun. Math. Phys. 150(3), 593-630 (1992)

[JW94] Jeffrey, L., Weitsman, J.: Toric structures on the moduli space of flat connections on a Riemann surface: volumes and the moment map. Adv. Math. 106(2), 151-168 (1994)

[KRWY] Kashaev, R., Reshetikhin, N., Webster, B., Yakimov, M.: Moduli Spaces of Flat $G$-connections and Symmetrically Factorizable Groups, Preprint

[K93] Kontsevich, M.: Vassiliev's knot invariants. In: Gelfand Seminar, I.M. (ed.) Advances in Soviet Mathematics, vol. 16, pp. 137-150. American Math Soc, Providence (1993)

[Law07] Lawton, S.: Generators, relations and symmetries in pairs of $3 \times 3$ unimodular matrices. J. Algebra 313(2), 782-801 (2007)

[Law09] Lawton, S.: Poisson geometry of $S L(3, \mathbb{C})$-character varieties relative to a surface with boundary. Trans. Am. Math. Soc. 361(5), 2397-2429 (2009)

[Meu06] Meusburger, C.: Dual generators of the fundamental group and the moduli space of flat connections. J. Phys. A Math. Gen. 39(47), 14781 (2006)

[MF78] Mishchenko, A.S., Fomenko, A.T.: Generalized Liouville method of integration of Hamiltonian systems. Funct. Anal. Appl. 12(2), 113-121 (1978)

[Nek72] Nekhoroshev, N.: Action-angle variables, and their generalizations. Trans. Mosc. Math. Soc. 26, 181-198 (1972)

[Nek96] Nekrasov, N.: Holomorphyc bundles and many-body systems. Commun. Math. Phys. 180, 587604 (1996)

[Pa26] Pauli, W.: On the hydrogen spectrum from the standpoint of the new quantum mechanics. Z. Phys. 36, 336-363 (1926)

[Res03a] Reshetikhin, N.: Integrability of characteristic Hamiltonian systems on simple Lie groups with standard Poisson Lie structure. Commun. Math. Phys. 242(1), 1-29 (2003)

[Res03b] Reshetikhin, N.: Degenerate integrability of the spin Calogero-Moser systems and the duality with the spin Ruijsenaars systems. Lett. Math. Phys. 63(1), 55-71 (2003)

[Res16] Reshetikhin, N.: Degenerately integrable systems. J. Math. Sci. 213(5), 769-785 (2016)

[Res18] Reshetikhin, N.: Spin Calogero-Moser models on symmetric spaces. preprint arXiv:1903.03685 
[RS19] Reshetikhin, N., Stokman, J.: Classical spin Calogero-Moser-Sutherland type systems and boundary Knizhnik-Zamolodchikov equation. To appear

[RT90] Reshetikhin, N., Turaev, V.: Ribbon graphs and their invaraints derived from quantum groups. Commun. Math. Phys. 127(1), 1-26 (1990)

[SS17] Schrader, G., Shapiro, A.: Continuous tensor categories from quantum groups I: algebraic aspects. preprint arXiv: 1708.08107

[SInt] Tempesta, P., Winternitz, P., Harnad, J., Miller, W., Jr., Pogosyan, G., Rodriguez, M. (eds.): Superintegrability in Classical and Quantum Systems. CRM Proceedings and Lecture Notes, vol. 37 (2004)

[T91] Turaev, V.: Skein quantization of Poisson algebras of loops on surfaces. Ann. Sci. l'Ecole Norm. Supérieure 24(6), 635-704 (1991)

[V90] Vassiliev, V.: Cohomology of knot spaces In: Theory of Singularities and Applications, Advances in Soviet Mathematics, vol. 1, pp. 23-69. American Math Soc, Providence (1990)

Communicated by H.-T. Yau 